\section{COMPORTAMIENTO DEL HORMIGON A MUY BAJAS TEMPERATURAS}

\author{
Prof. Manuel Elices Calafat*
}

689-13

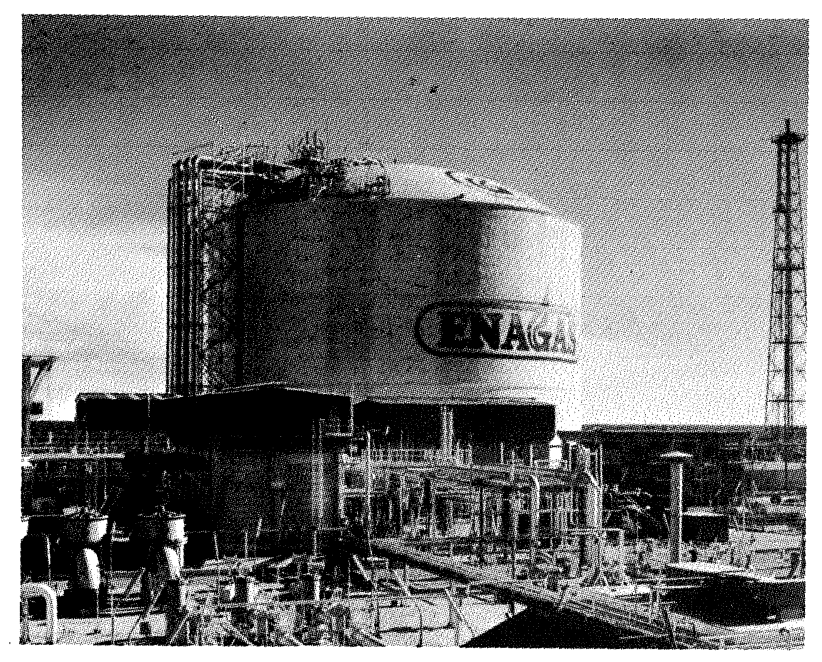

\section{Introducción}

\section{Hormigón}

2.1. Heladicidad del agua en los poros.

2.2. Resistencia a la compresión.

2.3. Resistencia a la tracción.

\section{Hormigón armado}

3.1. Deformación térmica.

3.2. Adherencia con el acero.

\section{Hormigón pretensado}

4.1. Fluencia del hormigón.

4.2. Conductividad térmica y calor específico.

\section{Conclusiones}

* Dpto. de Física y Física de Materiales,

E.T.S. de Ingenieros de Caminos,

Universidad Politécnica de Madrid

\section{1. - Introducción}

Este trabajo se refiere al comportamiento de hormigones fabricados y curados a temperatura ambiente y que posteriormente son enfriados. En estos casos, el proyectista utiliza sólo datos para temperatura ambiente y considera que el incremento de resistencia que puede producirse al enfriar redunda en una mayor seguridad. El constructor también sigue los métodos de control y ensayo diseñados para temperatura ambiente, entre otros motivos, porque no dispone de una metodologia y una normativa para estas circunstancias.

El interés por este campo nació durante la realización de una serie de estudios y ensayos, en el Departamento de Física y Física de Materiales de la Escuela de Ingenieros de Caminos, motivados por la construcción de un tanque de hormigón pretensado para almacenar $80.000 \mathrm{~m}^{3}$ de gas natural licuado. Este interés se despertó gracias a las inquietudes $y$ al celo de los promotores y constructores de esta obra singular: ENAGAS, S. A. y AUXINI, S. A.

Situaciones parecidas, y por desgracia escasas, se han dado durante los últimos años. Asi en USA se hicieron los primeros estudios por iniciativa conjunta de la American Gas Association y de la Portland Cement Association. (Artículos de Monfore y Lentz 1962, 1965, 1966). Posteriormente, gracias a los trabajos promovidos por Italcementi se clarificó algo más el campo, permitiendo eliminar los efectos de algunas variables (Tognon, 1969). Una tercera serie de ensayos, financiada por Shimizu Construction Co., estableció las primeras formulas predictivas para las propiedades más importantes (Okada, Iguro, 1978). Estas referencias, junto con los trabajos japoneses de $S$. Yamane $y$ el Prof. Y. Goto y los más recientes del Prof. F. Rostasy, constituyen la principal fuente de información de este artículo. En él se pretende dar una panorámica del estado actual del conocimiento sobre el comportamiento del hormigón a bajas temperaturas, con sus logros e interrogantes. Con objeto de evitar, en lo posible, la monotonia, las propiedades se han estructurado agrupándolas según su relación, más o menos directa, con el hormigón, el hormigón armado y el hormigón pretensado. Esta es la única razón de la división en apartados de este trabajo. 
El artículo va dirigido al proyectista, ofreciéndole datos para poder estimar la resistencia del hormigón y otros parámetros que entran en el cálculo, al constructor dándole criterios que le permitan decidir las variables que debe controlar para ello y al científico para que las numerosas lagunas detectadas le sirvan de estímulo.

\section{2.-Hormigón}

\subsection{Heladicidad del agua en los poros}

A lo largo de este estudio veremos que la humedad del hormigón desempeña un papel decisivo en su comportamiento a bajas temperaturas. En realidad, es el contenido de agua que puede helarse lo que influye en las propiedades mecánicas de este material. Por debajo de determinadas temperaturas no tenemos solamente un hormigón progresivamente más frio, sino un sistema formado por hielo y hormigón que exhibe un comportamiento sorprendente y ventajoso para algunas aplicaciones. Asi, por ejemplo, para ciertas temperaturas la resistencia a la compresión a temperatura ambiente puede triplicarse y lo mismo puede sucederle a la deformació.ı, debido a la gran resistencia del hielo y al micropretensado que crea el agua al helarse.

Para nuestro propósito podemos considerar al hormigón como un material compuesto por dos fases: los áridos y la pasta de cemento. La humedad del hormigón es, prácticamente, la de la pasta de cemento cuando se utilizan áridos compactos $y$, por consiguiente, el agua susceptible de helarse será la contenida en los poros del gel y en los poros capilares. A este agua hay que añadirle la de los poros macroscópicos (M. Elices, F. Rostasy, W. Faas, 1982, P. Grubl, 1980).

Durante el enfriamiento el agua se va transformando en hielo en distintas etapas ( $N$. Stockhausen, 1979): El agua almacenada en los huecos más grandes se va convirtiendo en hielo entre $0{ }^{\circ} \mathrm{C}$ y $-10{ }^{\circ} \mathrm{C}$, dependiendo de las substancias disueltas en ella. El agua condensada en los poros capilares se hiela entre $-30^{\circ} \mathrm{C}$ y $-80^{\circ} \mathrm{C}$. Finalmente, el agua absorbida en las paredes de los poros más pequeños puede helarse a $-160{ }^{\circ} \mathrm{C}$. (Fig. 1). Por consiguiente, al enfriar el hormigón la transición de fase es gradual, coexistiendo hielo, agua y vapor de agua en un amplio intervalo de temperaturas.

El efecto perjudicial en el hormigón de los ciclos hielo-deshielo es bien conocido. Se trata de un proceso complejo causado por fenómenos a nivel microscópico y macroscópico. Los primeros, debidos a la estructura de la pasta de cemento, se pueden atribuir al incremento de presión originado por el incremento de volumen del agua al helarse $(9 \%)$, cuando encuentra impedimentos para acomodarse y, también, a sobrepresiones diferencia-
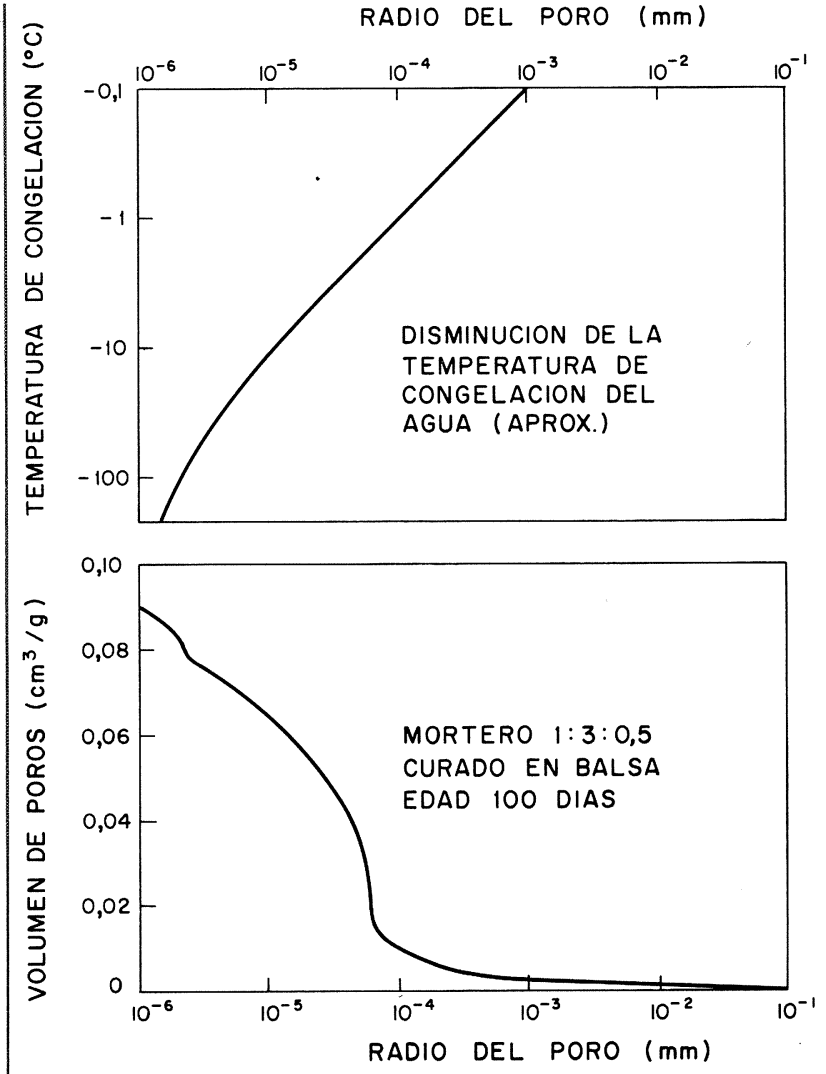

Fig. 1. - Temperatura de solidificación del agua en el hormigón en función del tamaño de los poros y distribución de los mismos en un mortero de cemento.

les originadas por la difusión del agua desde los pequeños poros hacia el hielo (M. J. Setzer, 1977). Ambos procesos causan microfisuración y pueden reducirse fabricando hormigones con la menor cantidad posible de agua que se pueda helar, utilizando para ello relaciones agua/cemento $<0,45$ y agentes aireantes.

A nivel macroscópico, los gradientes fuertes de temperatura ocasionan transitoriamente tensiones de origen térmico que pueden producir fisuras. Por ejemplo, si se crea bruscamente una zona fría (cold-spot) en la pared exterior de un tanque. La diferencia de coeficientes de dilatación entre los áridos y la pasta de cemento también puede ser el origen de fisuras. En consecuencia, deben evítarse los cambios bruscos de temperatura y es aconsejable que los materiales del hormigón sean compatibles térmicamente.

\subsection{Resistencia a la compresión}

El conocimiento que se posee sobre la resistencia del hormigón a bajas temperaturas proviene de los escasos ensayos uniaxiles realizados (el autor no conoce referencias sobre ensayos bi o triaxiles a temperaturas criogénicas, con excepción de los datos mencionados en el articulo de G. Tognon). En estas circunstancias, el comportamiento del 
hormigón se estima a partir de los ensayos uniaxiles siguiendo los mismos criterios que a temperatura ambiente.

Además, la falta de normalización del mencionado ensayo añade nuevas imprecisiones. Algunas variables del ensayo no parecen muy críticas dentro de los rangos usuales, por ejemplo: la velocidad de enfriamiento (entre 1 y $10^{\circ} \mathrm{C}$ por minuto), la velocidad de solicitación (entre $5 \times 10^{-3} \mathrm{~mm} / \mathrm{seg}$ y $5 \times 10^{-2} \mathrm{~mm} / \mathrm{seg}$ ), la edad (para probetas de más de dos meses) etc.; no obstante merece la pena estudiar su influencia. Existen otras variables más criticas, como las condiciones de curado y el contenido de agua que puede helarse. El control de la humedad durante los ensayos, especialmente si se estudia el comportamiento durante varios ciclos térmicos, es fundamental $(\mathrm{H}$. Corres, J. Planas, M. Elices, 1981).

A continuación se comenta la influencia de la temperatura, de la humedad y de los ciclos térmicos en las curvas tensión-deformación obtenidas a partir de ensayos de compresión con probetas cilindricas. Los resultados se han tomado de la comunicación del Prof. F. Rostasy, en el $1^{\text {er }}$ Congreso Internacional sobre Hormigón para bajas temperaturas (F. Rostasy, G. Wiedemann, 1981). Los ensayos se han realizado con hormigones fabricados con cemento portland, áridos silíceos y con las dosificaciones indicadas en las figuras 2, 3 y 4.

En la figura 2 se han representado diversos diagramas tensión-deformación para ensayos de compresión realizados desde temperatura ambiente hasta $-170{ }^{\circ} \mathrm{C}$, en probetas saturadas con agua. Merece destacarse que en el intervalo $20^{\circ} \mathrm{C}$, $-80{ }^{\circ} \mathrm{C}$ no sólo aumenta la resistencia sino que, además, aumenta la deformación. El hormigón en vez de volverse más frágil, como era de esperar intuitivamente, aumenta su ductilidad (medida como deformación bajo carga máxima). Para temperaturas inferiores, la relación tensión-deformación va perdiendo su carácter no lineal y se va transformando progresivamente en una recta. Las deformaciones bajo carga máxima empiezan a decrecer hasta recuperar los valores correspondientes a temperatura ambiente.

En la figura 3 se pone de manifiesto la influencia de la humedad. Las probetas se ensayaron a los 120 dias. Después de curadas, un lote se almacenó a $20{ }^{\circ} \mathrm{C}$ y con una humedad relativa del $65 \%$, $y$ el otro permaneció sumergido en agua. A temperatura ambiente las diferencias son pequeñas, pero a $-170{ }^{\circ} \mathrm{C}$ las probetas saturadas exhiben un comportamiento elástico-lineal mientras que las almacenadas en ambiente húmedo presentan un ligero carácter no lineal y una resistencia mucho menor.

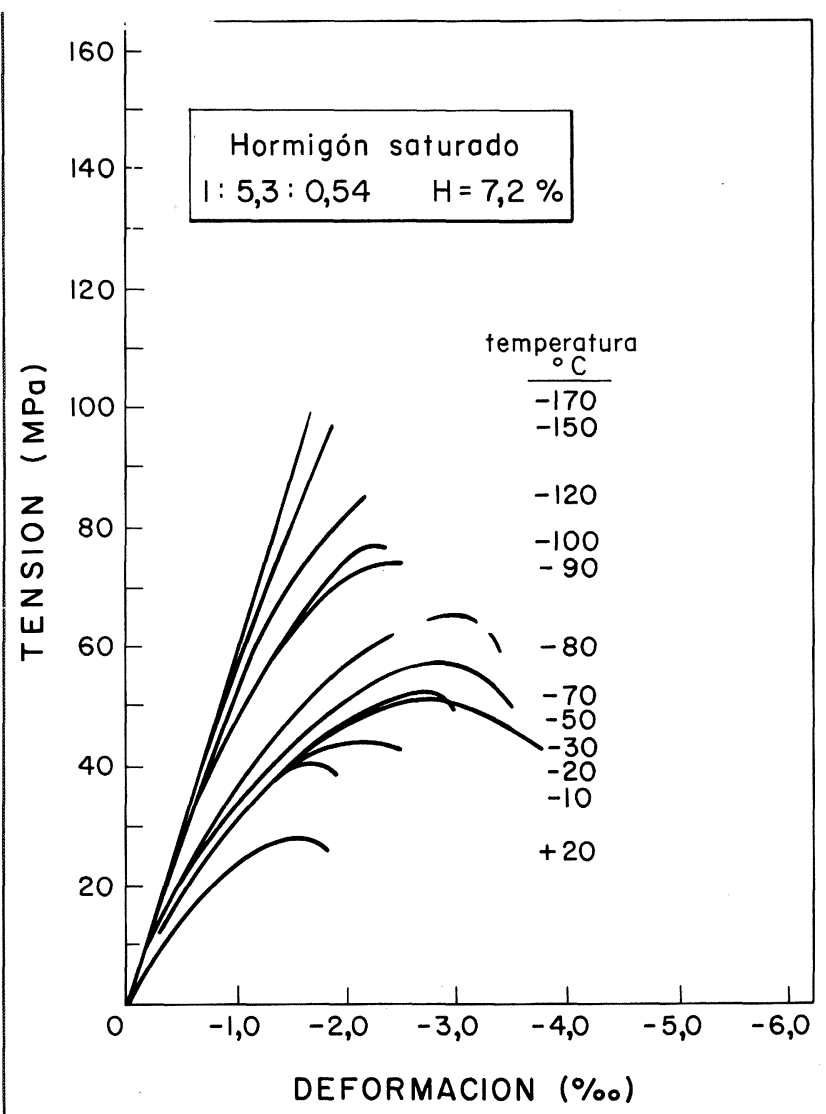

Fig. 2.--Ensayos de compresión. Influencia de la temperatura en la curva tensión-deformación.

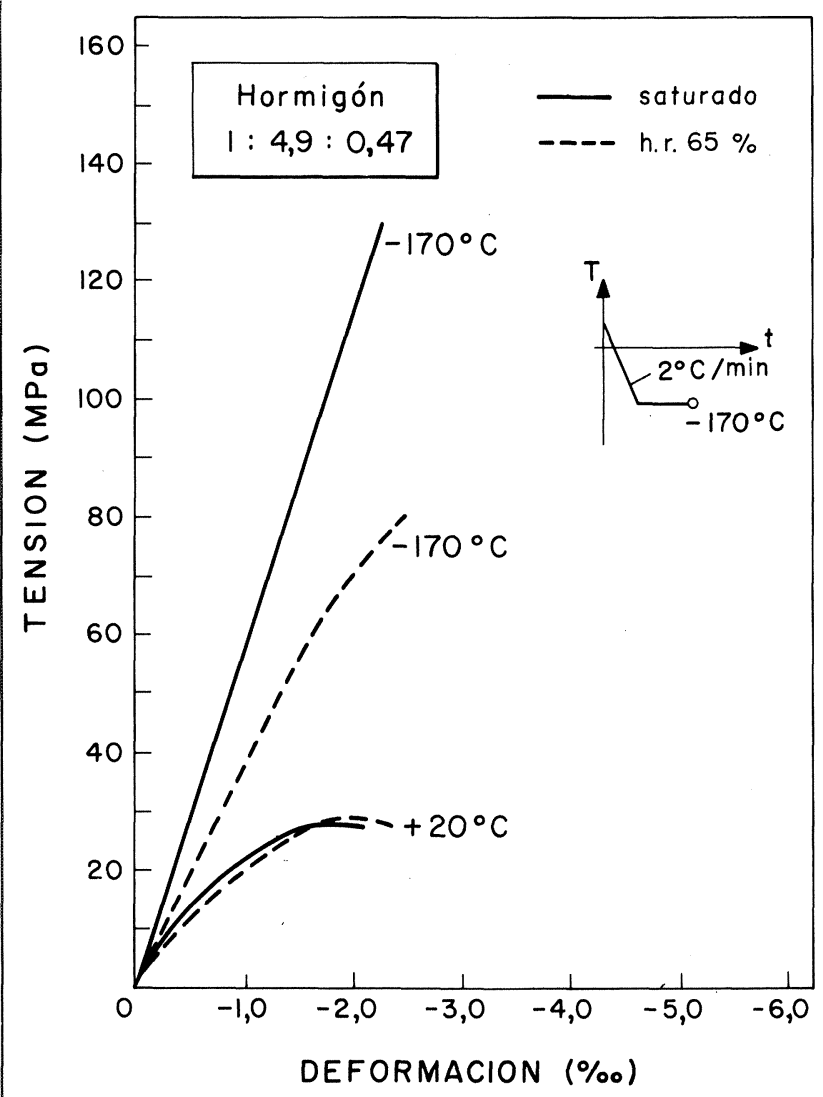

Fig. 3.--Ensayos de compresión. Influencia de la humedad en la curva tensión-deformación. 
En la figura 4 se puede observar la influencia de los ciclos térmicos en la forma de las curvas tensión-deformación. Como puede apreciarse, tanto la resistencia como el módulo de elasticidad disminuyen apreciablemente con unos pocos ciclos. La forma de realización del ensayo se indica esquemáticamente en la figura. Las probetas se han ensayado saturadas. También se ha comprobado que el módulo de elasticidad disminuye a medida que aumenta la relación agua/cemento en el intervalo 0,50-0,65. Esta pérdida en propiedades mecánicas puede subsanarse en gran medida mediante la utilización de hormigones microrreforzados con fibras de acero (F. Rostasy, G. Wiedemann, 1981).

El dato que más interesa al proyectista es, casi siempre, la resistencia a la compresión. Por lo que se acaba de ver, cabe esperar que dicho parámetro tenga una fuerte dependencia de la humedad, además de la relación agua/cemento, dosificación, edad y otras variables que influyen a temperatura ambiente.

En la figura 5 se han representado los valores relativos de la resistencia a la compresión (referidos al valor de la temperatura ambiente) en función de la temperatura para distintos valores de la humedad. Para hormigones saturados es de destacar que su resistencia puede aumentar en un $400 \%$, a temperaturas muy bajas. El aumento de resistencia es menos importante a medida que la hu-

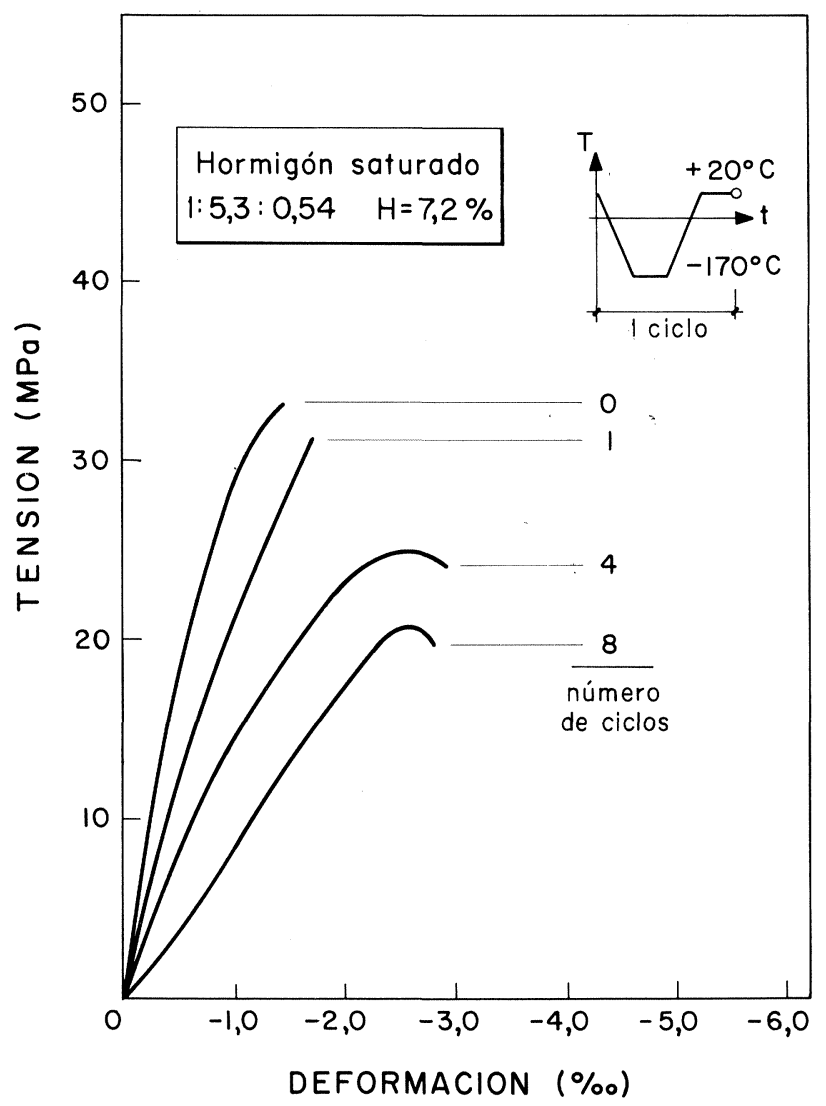

Fig. 4. Influencia de los ciclos térmicos en la curva tensión-deformación. medad disminuye pero, incluso para valores del $2 \%$, es notable. Este fenómeno puede atribuirse a la gran resistencia del hielo y a que al helarse completamente los poros capilares no actúan como fisuras, concentrando tensiones e iniciando la microfisuración. La tendencia de las curvas de la figura 5, tomadas de la citada comunicación de F. Rostasy es similar a las ya publicadas por Montfore (1962), Tognon (1969), Okada (1978), Yamane (1978) y Goto (1979).

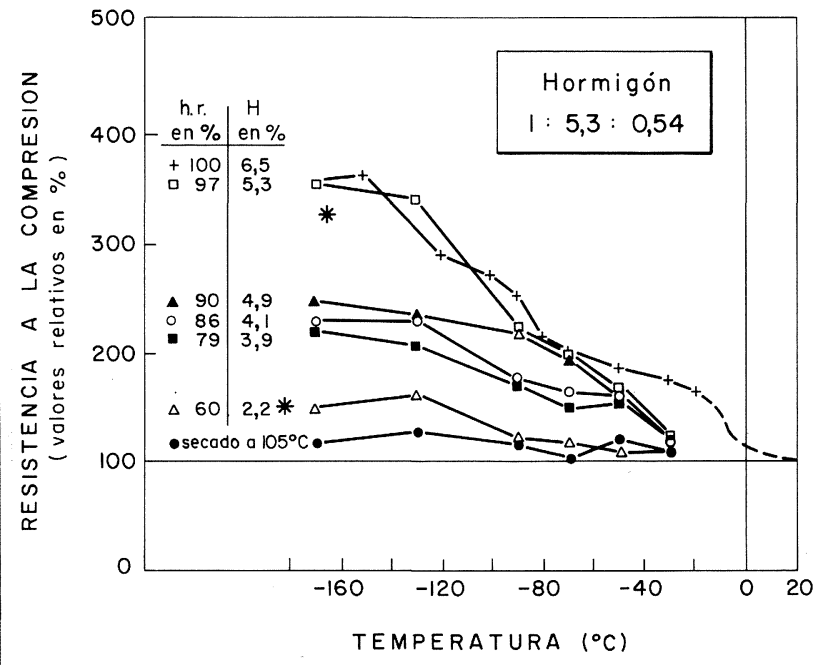

Fig. 5. --Resistencia a compresión del hormigón en función de la temperatura. Influencia de la humedad.

La pérdida de resistencia debida a ciclos térmicos se ha reflejado en la figura 6 . ( $F$. Rostasy et al. 1979). El deterioro del hormigón aumenta con el número de ciclos. Para el mismo número de ciclos, también aumenta al disminuir la temperatura de enfriamiento hasta temperaturas alrededor de $-70{ }^{\circ} \mathrm{C}$. A partir de estas temperaturas la disminución de resistencia es mucho menor y parece que está ligada con la deformación térmica, como veremos más adelante. Alrededor de $-70{ }^{\circ} \mathrm{C}$ la mayor parte del agua se ha transformado en hielo, habiéndose producido ya los daños ocasionados por el incremento de volumen. Ensayos similares realizados con probetas almacenadas con una humedad del $65 \%$ apenás detectaron pérdidas de resistencia (figura 6), confirmando nuevamente el papel decisivo que juega la humedad a bajas temperaturas.

Para calcular la resistencia a la compresión varios autores han propuesto relaciones empiricas, ajustando funciones sencillas a un conjunto de datos experimentales. Estas expresiones permiten calcular la resistencia a una temperatura dada, $\sigma_{c}(T)$, en función de la resistencia a temperatura ambiente, $\sigma_{c}\left(T_{R}\right)$, de la temperatura, $T, y$, en algunos casos, de la humedad $H$. Algunas de las relaciones propuestas se indican a continuación: 


$$
\begin{aligned}
& \sigma_{c}(\mathrm{~T})=\sigma_{c}\left(\mathrm{~T}_{R}\right)+5,3-0,84 \mathrm{~T}-0,0027 \mathrm{~T}^{2} \\
& \left(-10^{\circ} \mathrm{C}>\mathrm{T}>-100^{\circ} \mathrm{C}\right)(\text { Okada, 78) } \\
& \sigma_{c}(\mathrm{~T})=\sigma_{c}\left(\mathrm{~T}_{R}\right)-(2 / 15+\mathrm{T} / 2700) \mathrm{TH} \\
& \left(0^{\circ} \mathrm{C}>\mathrm{T}>-120^{\circ} \mathrm{C}\right)(\text { Goto, } 79)
\end{aligned}
$$

$$
\begin{aligned}
& \sigma_{c}(\mathrm{~T})=\sigma_{c}\left(\mathrm{~T}_{R}\right)-\mathrm{TH} / 12 \\
& \left(0^{\circ} \mathrm{C}>\mathrm{T}>-120^{\circ} \mathrm{C}\right)(\text { Browne, 81) }
\end{aligned}
$$

donde la resistencia está expresada en $N / \mathrm{mm}^{2}$ y $H$ es la humedad del hormigón, en \%. A partir de $-120^{\circ} \mathrm{C}$, aproximadamente, la resistencia crece muy lentamente $y$, como la dispersión de los resultados es grande, es aconsejable suponer que la resistencia permanece constante. En la figura 7 se han representado las expresiones anteriores, junto con algunos resultados experimentales obtenidos en la Escuela de Ingenieros de Caminos.

Finalmente, sería deseable conocer la influencia de los áridos, tipo de cemento y dosificación del

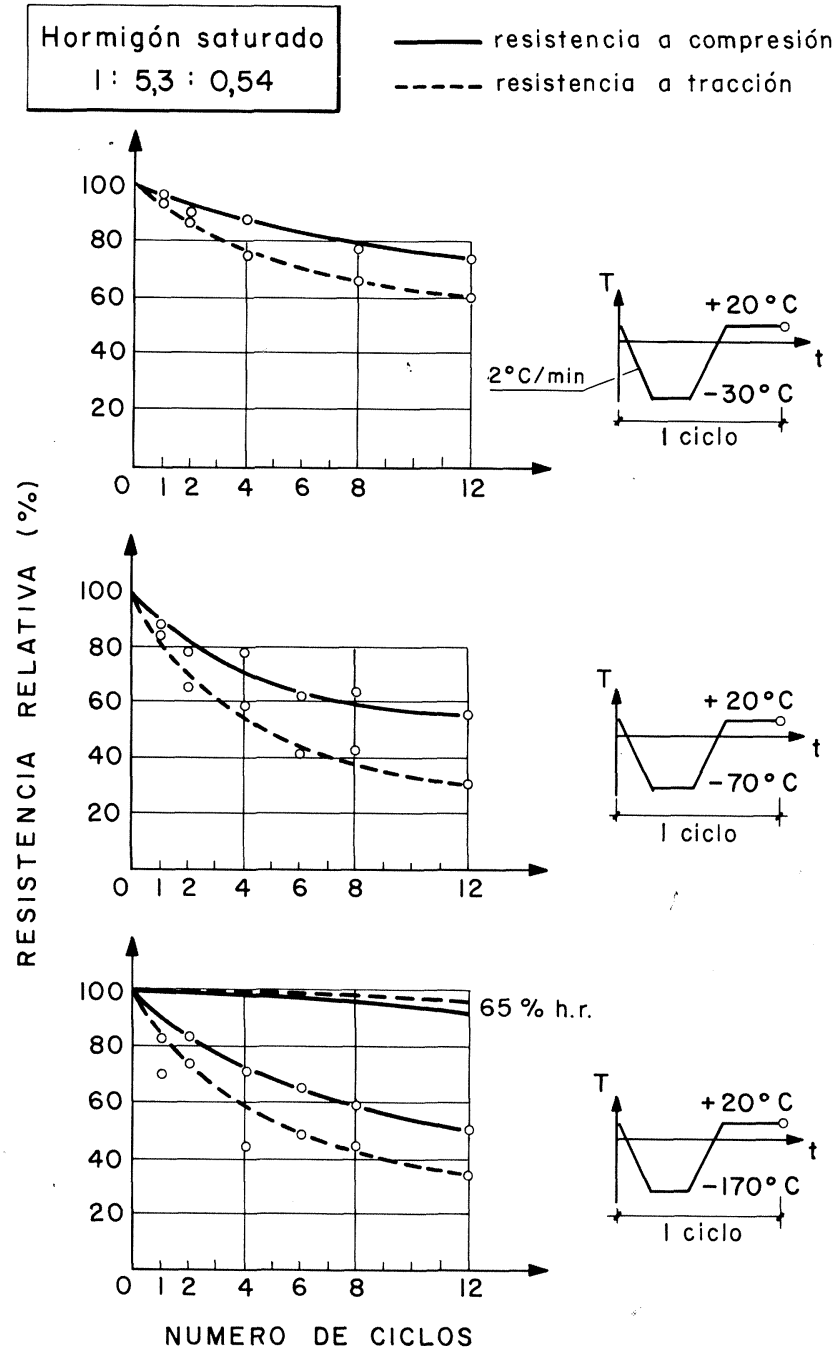

Fig. 6.--Resistencia del hormigón después de varios ciclos térmicos. Influencia de la temperatura y de la humedad.

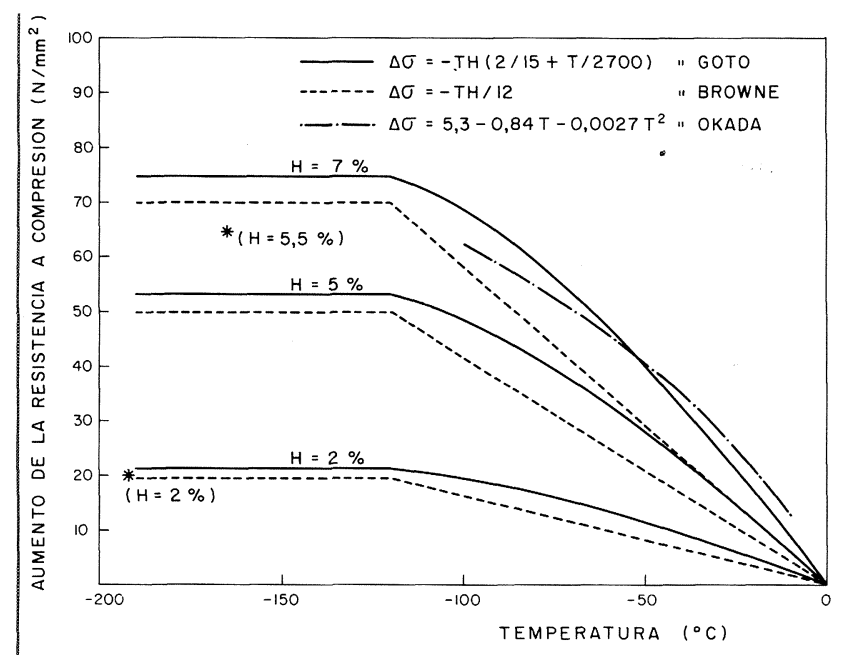

Fig. 7.--Expresiones propuestas para estimar la resistencia a compresión a bajas temperaturas. *(Resultados experimentales obtenidos en la Escuela de Caminos).

hormigón, en la resistencia a bajas temperaturas. Aunque estas propiedades no se han estudiado sistemáticamente ya se dispone de algunos resultados orientativos. En general, si exceptuamos la humedad, las otras variables no parece que tengan una incidencia a bajas temperaturas mayor de la que se observa a temperatura ambiente. La influencia de algunos tipos de cemento (portland, puzolánico, escorias de altos hornos y cemento rápido) puede verse en los trabajos de G. Tognon (1969), F. Rostasy (1979) y Y. Goto (1979). La influencia de los áridos también la ha considerado G. Tognon (1969). El efecto de la relación agua/ cemento puede verse en los trabajos citados de F. Rostasy y en el artículo de S. Yamane (1978), y la influencia de los aireantes se comenta en los trabajos de S. Yamane (1978) y Y. Goto (1979).

\subsection{Resistencia a la tracción}

En el hormigón la resistencia a tracción se estima mediante ensayos indirectos; flexotracción y el ensayo Brasileño. La falta de normalización - para bajas temperaturas - de estos ensayos y la escasez de detalles en la descripción de los pocos ensayos realizados dificultan la interpretación de los resultados disponibles.

Recientemente se ha propuesto otro ensayo para bajas temperaturas, el ensayo de punzonamiento doble (M. Elices y J. Planas, 1982), cuya realización es más sencilla y los resultados parecen ser más fiables al no preseleccionar el plano de rotura. Además, se puede llevar a cabo en una máquina de compresión de menor capacidad y permite utilizar probetas con la misma geometria que para el ensayo de compresión.

En la figura 8 se ha representado esquemáticamente el aumento de resistencia a tracción en función de la temperatura y para distintos valores de la humedad. Esta figura, tomada del artículo 
de Browne y Bamforth (1981), está basada en los resultados de Montfore (1962), Eakin (1963), Okada (1978) y Yamane (1978). La resistencia disminuye al disminuir la humedad y aumenta con la disminución de la temperatura. A partir de $-100^{\circ} \mathrm{C}$, aproximadamente, tiende a disminuir en vez de estabilizarse, a diferencia de la resistencia a la compresión.

La pérdida de resistencia después de varios ciclos térmicos se ha indicado en la figura 6 . La tendencia de los resultados es similar a los obtenidos para la resistencia a la compresión comentados anteriormente.

La resistencia a tracción puede estimarse a partir de expresiones obtenidas ajustando los resultados experimentales. Se han propuesto las siguientes fórmulas, donde la resistencia a tracción, $\sigma_{t}(T)$ (en $\mathrm{N} / \mathrm{mm}^{2}$ ) a una temperatura $T$, viene en función de la resistencia a compresión a la misma temperatura, $\sigma_{c}(\mathrm{~T})$ :
$\sigma_{t}(T)=2,4+0,06 \sigma_{c}(T) \quad$ (Okada, 1978)
$\sigma_{t}(T)=0,214 \sigma_{c}{ }^{0,75}(T)$ (Goto, 1979)

Los valores propuestos por Okada son válidos para el intervalo $-10^{\circ} \mathrm{C}$ hasta $-100{ }^{\circ} \mathrm{C}$, aunque pueden utilizarse para temperaturas más bajas, como sucede para la resistencia a la compresión. Los valores de Goto son válidos hasta $-160^{\circ} \mathrm{C}$, por lo menos, y para hormigones con relaciones agua/cemento entre 0,45 y 0,55 . La correlación entre la resistencia a tracción $\sigma_{t}$ y la resistencia a flexotracción $\sigma_{f t}$, a bajas temperaturas, es, aproximadamente, $\quad \sigma_{f t}=2,0 \quad \sigma_{t} ;$ a temperatura ambiente dicha correlación es ligeramente inferior.

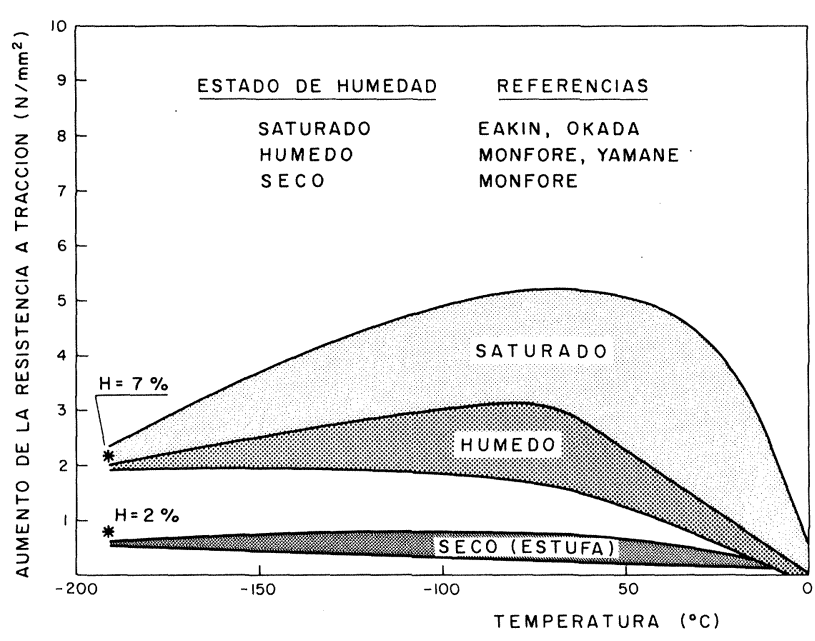

Fig. 8. --Aumento de la resistencia a tracción en función de la temperatura y para distintos valores de la humedad.

Como resumen de lo expuesto, en la Tabla 1 se han incluido algunos resultados de los ensayos realizados en el Departamento de Física y Física de Materiales de la Escuela de Caminos y se han comparado con las predicciones teóricas.

Los resultados de la resistencia a compresión, para humedades intermedias, coinciden bastante bien con las predicciones. (Para la fórmula de Okada se ha tomado $\mathrm{T}=-100^{\circ} \mathrm{C}$. El valor de Monfore es ligeramente superior por corresponder a probetas con un contenido de humedad mayor). Para probetas casi secas, $\mathrm{H}=2 \%$, la fórmula de Goto da resultados satisfactorios, mientras que la de Okada, que no tiene en cuenta la humedad, sobreestima la resistencia. (Los valores de Monfore son inferiores porque corresponden a probetas desecadas). Los valores experimentales de la resistencia a la compresión a temperatura ambiente son: $32,6 \mathrm{~N} / \mathrm{mm}^{2}$ (28 dias) y $34,3 \mathrm{~N} / \mathrm{mm}^{2} \quad$ (22 meses).

Las predicciones para la resistencia a la tracción son bastante aproximadas. Nuevamente vemos que la fórmula de Okada sobreestima la resistencia cuando la humedad es baja y parece que ocurre lo mismo con la de Goto cuando la humedad es alta. Los valores experimentales están dentro del intervalo marcado por los valores extremos de Monfore. La resistencia a la compresión a -196 grados centigrados, para las probetas con $2 \%$ de humedad, es $53,5 \mathrm{~N} / \mathrm{mm}^{2}$. No se tienen datos para las probetas con $7 \%$ de humedad. El valor de Goto se ha estimado a partir del dato 53,5 $\mathrm{N} / \mathrm{mm}^{2}$.

Otros datos de interés para el proyectista son: el módulo de elasticidad y el coeficiente de Poisson. T. Okada y $\mathrm{M}$. Iguro han propuesto las siguientes expresiones para el intervalo $-10^{\circ} \mathrm{C},-100^{\circ} \mathrm{C}$;

$E(T)=300 \sigma_{c}(T)+1,33 \times 10^{10}($ Okada, 1978) $v=0,23$

donde $E(T)$ y $\sigma_{c}(T)$ vienen dados en $N / m^{2}$ y repre sentan, respectivamente, el módulo de elasticidad y la resistencia a la compresión a la temperatura $T$. El coeficiente de Poisson permanece más o menos constante con la variación de temperatura y se han publicado valores entre 0,19 y 0,27.

Quedan todavia otros aspectos del comportamiento mecánico del hormigón a bajas temperaturas, de enorme interés para el proyectista, que aún no se han explorado. Es interesante conocer la respuesta del hormigón frio frente a cargas aplicadas bruscamente, o del hormigón, a temperatura ambiente, enfriado súbitamente. Ambos tipos de solicitación se pueden dar en un accidente y conviene conocer la capacidad resistente de la estructura bajo estas acciones. Se han publicado muy pocos trabajos en esta dirección y los datos de que se dispone son fragmentarios y no permiten sacar conclusiones definitivas que sean útiles al proyectista. 
TABLA 1. RESISTENCIA DEL HORMIGON A BAJAS TEMPERATURAS ( $/ \mathbf{~ m m}^{2}$ )

\begin{tabular}{|c|c|c|c|c|}
\hline \multirow[b]{2}{*}{$\begin{array}{l}\text { Edad } \\
\text { Temperatura } \\
\text { Humedad }\end{array}$} & \multicolumn{2}{|c|}{ RESISTENCIA A COMPRESION } & \multicolumn{2}{|c|}{ RESISTENCIA A TRACCION } \\
\hline & $\begin{array}{c}28 \text { dias } \\
-165{ }^{\circ} \mathrm{C} \\
5,5 \%\end{array}$ & $\begin{array}{c}22 \text { meses } \\
-196{ }^{\circ} \mathrm{C} \\
2 \%\end{array}$ & $\begin{array}{c}22 \text { meses } \\
-196{ }^{\circ} \mathrm{C} \\
7 \%\end{array}$ & $\begin{array}{c}22 \text { meses } \\
-196{ }^{\circ} \mathrm{C} \\
2 \%\end{array}$ \\
\hline $\begin{array}{l}\text { E. Caminos } \\
\text { Goto, Miura } \\
\text { Okada, Iguro } \\
\text { Monfore, Lentz }\end{array}$ & $\begin{array}{c}98,6 \\
91,4 \\
94,9 \\
108,0(h)\end{array}$ & $\begin{array}{l}53,5 \\
55,7 \\
96,6 \\
40,0(s)\end{array}$ & $\begin{array}{l}5,8 \\
7,2 \\
5,6 \\
6,0(h)\end{array}$ & $\begin{array}{l}5,0 \\
4,2 \\
5,6 \\
5,0 / s)\end{array}$ \\
\hline
\end{tabular}

(h) saturado; (s) seco

\section{3.-Hormigón armado}

En el hormigón armado el acero trabaja en colaboración con el hormigón. Cada material soporta aquellos esfuerzos que resiste mejor y transfiere los demás al otro. Cabe preguntarse si esta compatibilidad y capacidad de transferencia, esta armonia en suma, se mantendrá a bajas temperaturas.

Ambos materiales mejoran sus propiedades, no todas, a medida que disminuye la temperatura. Hemos visto que el hormigón incrementa su resistencia a la compresión y a la tracción, y que también aumenta su módulo de elasticidad. Todas ellas son muy sensibles al grado de humedad. Lo mismo ocurre con la fatiga térmica, aunque esta vez el incremento no sea deseable. Las propiedades de los aceros a bajas temperaturas, en particular de los aceros de armar, se han descrito en una monografia de la FIP (M. Elices, F. Rostasy y F. Faas, 1982). Tanto la resistencia como el módulo de elasticidad aumentan aunque no de forma tan espectacular como en el hormigón, y la ductilidad disminuye al disminuir la temperatura. En general, puede decirse que el comportamiento de acero a bajas temperaturas y para solicitaciones de servicio es satisfactorio. Cuando se producen concentraciones de tensiones y las solicitaciones son rápidas algunos aceros exhiben una fragilidad inadmisible para el proyectista. Para estas circunstancias se han desarrollado aceros de armar especiales cuyo comportamiento es aceptable (M. Elices, G. Sleigh, 1978).

Como ya se ha apuntado, no es suficiente con que los materiales se comporten satisfactoriamente, es el conjunto - el hormigón armado- el que debe ser adecuado. Es preciso que ambos materiales mantengan la compatibilidad al disminuir la temperatura; ello depende de cómo varien sus coeficientes de dilatación. En casos extremos, debido a que el hormigón se contrae menos que el acero, el hormigón puede introducir tensiones en las armaduras capaces de romperlas. Dedicaremos un apartado al estudio de la deformación térmica del hormigón. Además, la cooperación entre ambos materiales - la capacidad de transferencia de cargas, intimamente relacionado con la adherencia-, también merece explorarse a bajas temperaturas. A ello dedicaremos el segundo apartado.

\subsection{Deformación térmica}

EI conocimiento de las deformaciones de origen térmico es imprescindible para proyectar estructuras que van a estar sometidas a grandes variaciones de temperatura. Antes de analizar el coeficiente de dilatación empezaremos revisando cómo se deforma el hormigón al disminuir la temperatura.

En la figura 9 se comparan la deformación del acero y las de dos tipos de hormigón. Vemos que el acero y un hormigón, curado y almacenado en condiciones normales, muestran un comportamiento casi lineal y reversible (dependiendo la pendiente de la curva del hormigón del tipo de árido). Por el contrario, el hormigón saturado exhibe un comportamiento no lineal e irreversible, pudiéndose distinguir tres regiones:

a) Entre $20{ }^{\circ} \mathrm{C} \mathrm{y}-15{ }^{\circ} \mathrm{C}$, aproximadamente, el hormigón se contrae.

b) Entre $-20{ }^{\circ} \mathrm{C}$ y $-60{ }^{\circ} \mathrm{C}$, aproximadamente, se produce una expansión. A esta región la llamaremos región de transición. Este comportamiento se puede explicar teniendo en cuenta la transformación del agua en hielo en los pequeños poros que, como ya se indicó (figura 1), se produce gradualmente, dependiendo del tamaño de los mismos y de la concentración de las substancias disueltas.

c) Por debajo de $-70^{\circ} \mathrm{C}$, más o menos, el hormigón se contrae de nuevo.

La dilatación del hormigón depende de la velocidad de enfriamiento. La expansión en la región de transición aumenta al enfriar más de prisa. Por ejemplo; para una velocidad de $1^{\circ} \mathrm{C}$ por minuto se ha medido en morteros de cemento una expan- 
sión máxima del 0,3 por mil, alrededor de $-60^{\circ} \mathrm{C}$, mientras que para $10^{\circ} \mathrm{C}$ por minuto la expansión se duplica (M. Elices, F. Rostasy, W. Faas, 1982). Este fenómeno parece ser que se debe a que la congelación acelerada dificulta la reacomodación del agua, generando fuertes presiones. Las deformaciones irreversibles también aumentan con la velocidad de enfriamiento.

La dilatación en la zona de transición aumenta con la relación agua/cemento, ya que la cantidad de agua susceptible de helarse aumenta al incrementarse dicha relación. Por ejemplo, para probetas saturadas, un hormigón con $\mathrm{a} / \mathrm{c}=0,55$ puede alcanzar una deformación de un 0,2 por mil, mientras que con a/c $=0,70$ se llega al 0,7 por mil.

Al calentar una probeta previamente enfriada se observa, en determinadas condiciones, en la zona de transición una expansión todavia mayor (figura 9) y una deformación remanente (figura 9) que pone de manifiesto una microfisuración producida por el hielo. Cabe esperar que después de una serie de ciclos térmicos estos efectos se incrementen, y asi sucede. Algunos resultados de experimentos realizados con morteros saturados se muestran en la tabla 2. (F. Rostasy y G. Wiedemann, 1981).

La dilatación máxima, al enfriar, se produce alrededor de $-60^{\circ} \mathrm{C}$, mientras que la máxima expansión al calentar aparece alrededor de $-20^{\circ} \mathrm{C}$. Los ciclos se llevaron a cabo entre temperatura ambiente y $-110^{\circ} \mathrm{C}$, a una velocidad de enfriamiento de $2,5^{\circ} \mathrm{C}$ por minuto.

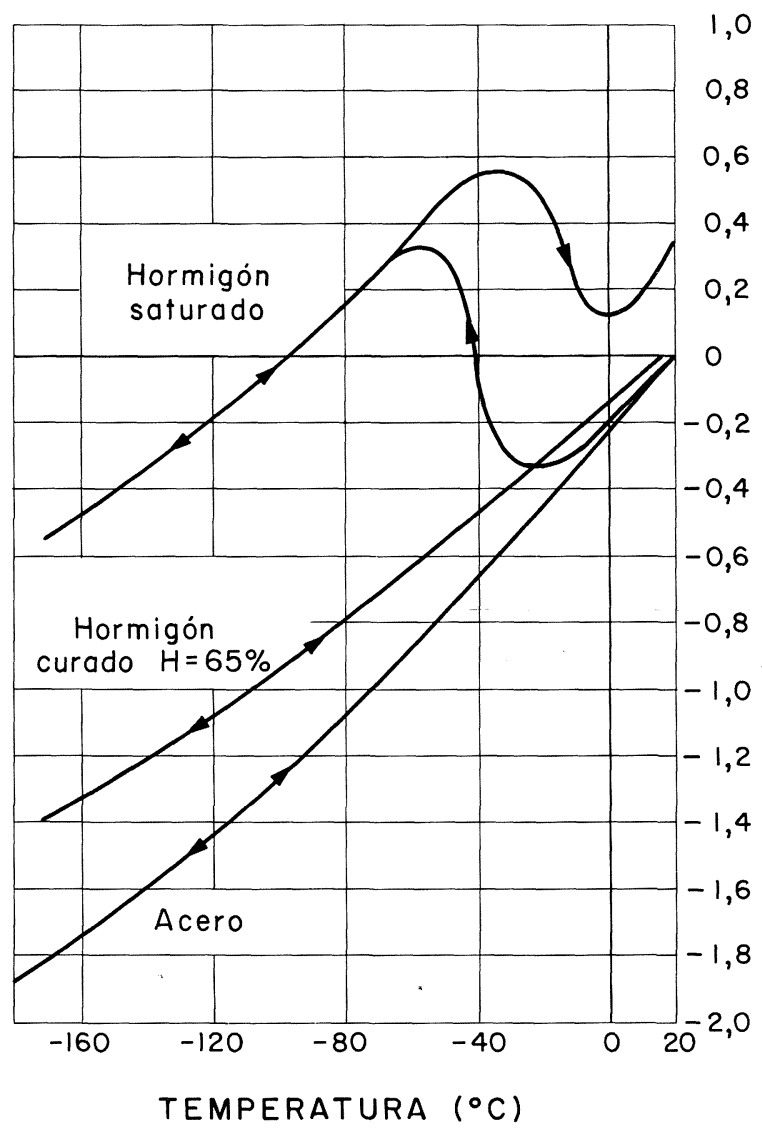

Fig. 9. --Dilatación del acero y del hormigón.

TABLA 2. INFLUENCIA DE LOS CICLOS TERMICOS EN LA DILATACION

\begin{tabular}{lcccc}
\hline \multicolumn{1}{c}{ Mortero saturado 1:3:0,5 } & 1.r Ciclo & 3.r Ciclo & 6. ${ }^{\circ}$ Ciclo & 12. $^{\circ}$ Ciclo \\
\hline Expansión máxima al enfriar $(\% \%)$ & 1,2 & 2,4 & 3,0 & 3,7 \\
Expansión máxima al calentar $(\% \%)$ & 1,6 & 2,6 & 3,1 & 3,9 \\
Deformación remanente $(\% \%)$ & 0,4 & 1,2 & 1,9 & 2,7 \\
\hline
\end{tabular}

Por último veamos cómo influye la humedad en la deformación térmica. Si permitimos que las probetas se sequen antes de enfriarlas, la cantidad de agua que puede helarse disminuirá $y$, por consiguiente, también serán menores la expansión en la región de transición y la deformación remanente. En la figura 10 se ha representado la deformación en función de la temperatura, para distintos grados de humedad. Las probetas se han curado y almacenado, durante seis meses, en ambientes con la humedad indicada en la figura. Un primer resultado que se observa inmediatamente es que las dilataciones en la región de transición aparecen sólo para humedades por encima del $86 \%$ y que cuando se cura al $50 \%$ de humedad relativa el agua que queda en el hormigón prácticamente no se hiela. Estos resultados conviene tenerlos presentes cuando se proyectan depósitos con camisa de chapa, ya que la humedad en los alrededores de dicha camisa puede ser del $97 \%$ o superior (F. Rostasy y G. Wiedemann, 1981).

Los fenómenos descritos anteriormente también están influenciados por la finura y el tipo de cemento utilizado. Se ha detectado que la expansión en la región de transición aumenta con la finura del cemento y que ensayando probetas con dos cementos de la misma finura, cemento portland y cemento de escorias, las probetas realizadas con este último dilatan más. Ya se ha mencionado 


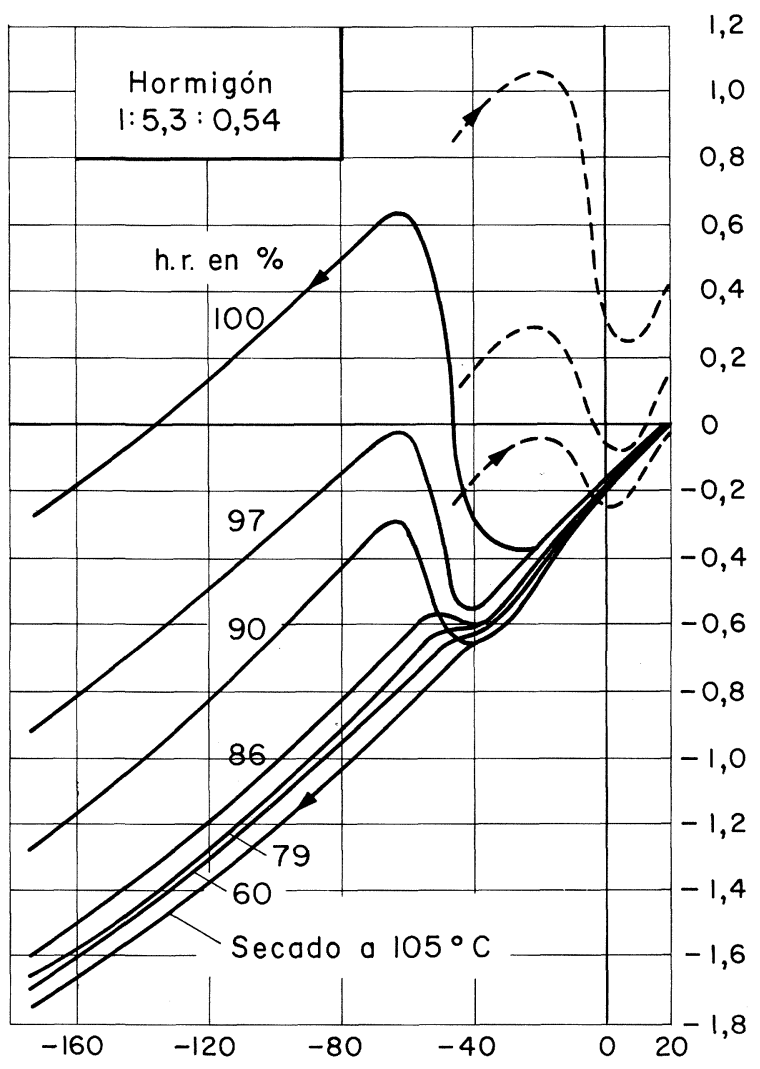

TEMPERATURA $\left({ }^{\circ} \mathrm{C}\right)$

Fig. 10.--Influencia de la humedad en la dilatación del hormigón.

que los áridos también influyen. Los áridos silíceos dan mayores deformaciones que los calcáreos.

Después de este resumen sobre la deformación del hormigón, donde hemos detectado que no se deforma como el acero, que podemos distinguir en función de la temperatura tres regiones de deformación y que la humedad es un factor decisivo, consideremos el coeficiente de dilatación, parámetro que interesa más al proyectista. El coeficiente de dilatación de los materiales es necesario conocerlo para poder estimar las tensiones internas producidas por las diferencias entre las deformaciones térmicas diferenciales de cada uno de los materiales. También es necesario saber el valor del coeficiente de dilatación del conjunto (hormigón armado o pretensado) cuando hay que calcular una estructura hiperestática con coacciones o la distribución de temperaturas y las tensiones de origen térmico.

Para temperaturas moderadamente bajas, hasta unos $-20^{\circ} \mathrm{C}$, el coeficiente de dilatación del acero y de los hormigones no difiere mucho, por eso, en la práctica, se utilizan valores comprendidos entre 10 y $12 \times 10^{-6} /{ }^{\circ} \mathrm{C}$. Las variaciones en el coeficiente de dilatación del hormigón están dominadas por la naturaleza de los áridos (valores altos para áridos siliceos y más bajos para calizos) y apenas se nota la influencia de la humedad.
Al disminuir la temperatura la humedad cobra importancia. Es costumbre utilizar un coeficiente de dilatación medio, definido por:

$$
\alpha(T)=\frac{\varepsilon(T)}{T-20}
$$

donde $\varepsilon(T)$ es la deformación a la temperatura $\mathrm{T}\left({ }^{\circ} \mathrm{C}\right)$, en vez del coeficiente tangente, definido por la pendiente de la curva $\varepsilon(T)$. En la figura 11 se ha representado esquemáticamente la variación del coeficiente de dilatación (medio) en función de la temperatura para hormigones con distinto contenido en humedad, junto con el coeficiente de dilatación de los aceros de construcción. Los hormigones con humedades altas reflejan el comportamiento de la dilatación que acabamos de comentar. Los hormigones con humedades alrededor del $85 \%$, e inferiores, exhiben siempre un coeficiente de dilatación positivo. Los hormigones con alto contenido de humedad, o saturados, pueden presentar coeficientes negativos (expansión, en vez de contracción) en un intervalo de temperatura. Esta expansión puede llegar a alcanzar, en valor absoluto, $32 \times 10^{-6} /{ }^{\circ} \mathrm{C}$. En estas condiciones se pueden generar a bajas temperaturas, tensiones
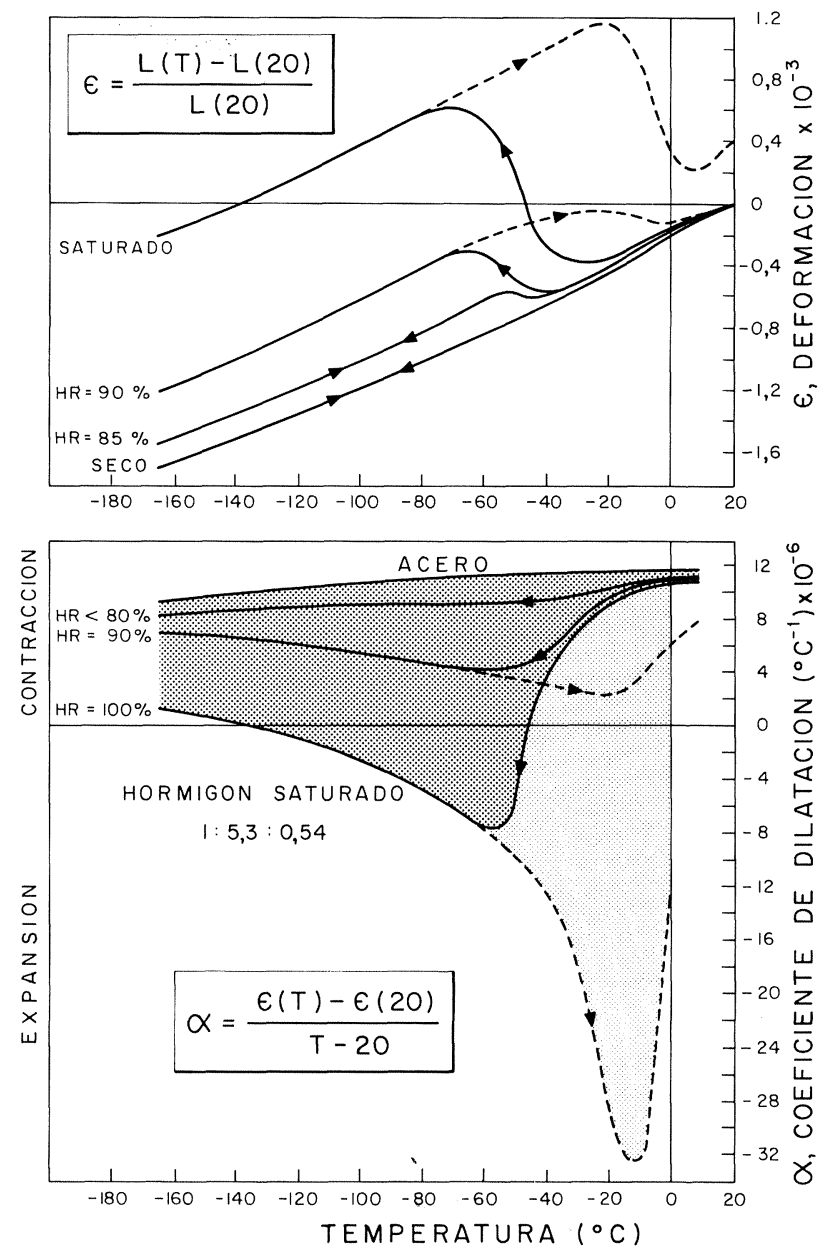

Fig. 11.--Regiones de los posibles valores del coeficiente de dilatación del hormigón en función de la temperatura y de la humedad. 
Internas del orden de $350 \mathrm{~N} / \mathrm{mm}^{2}$ capaces de romper algunas armaduras.

Los mismos comentarios que hemos hecho para la deformación en función de la temperatura son aplicables al coeficiente de dilatación. Este coeficiente depende de muchos parámetros y de la historia térmica, aunque la humedad sea el parámetro preponderante, y por ello no es posible, actualmente, dar curvas de carácter general para calcularlo. En cada caso deberán hacerse los ensayos pertinentes con los materiales y dosificación elegidos $y$ en las condiciones que simulen más acertadamente el comportamiento real. Tampoco hay ensayos normalizados para medir dicho coeficiente y algunas de las técnicas utilizadas para medirlo se han propuesto para su normalización (M. Elices, F. Rostasy y W. Faas, 1982). Uno de los muchos problemas que se encuentran para medir pequeñas deformaciones en probetas grandes de hormigón armado, o pretensado, es la estabilidad de los extensómetros. Recientemente se ha desarrollado un extensómetro (M. Elices, 1981), con una concepción distinta a los tradicionales, basado en una cavidad resonante de microondas que permite medir deformaciones del orden de $10^{-5}$ con un $10 \%$ de error, a distintas temperaturas y con una gran estabilidad que hace posible dejarlo embebido en el elemento estructural (Besada, J. L., Elices, M., Planas, J., Sánchez-Miñana, J., García, J. A., 1980).

\subsection{Adherencia con el acero}

Tanto para el dimensionamiento de los anclajes como para controlar la fisuración, el proyectista debe conocer los valores de la adherencia y la relación tensión de adherencia-desplazamiento para las temperaturas de trabajo. Asi como para temperatura ambiente estos fenómenos están bien documentados gracias a una importante experimentación; a bajas temperaturas sólo se dispone de dos series de ensayos, realizados por S. Yamane (1978) y Y. Goto (1979), que constituyen la fuente del siguiente resumen.

Algunos aspectos de la fisuración los han estudiado $Y$. Goto y T. Miura, sometiendo a tracción armaduras recubiertas de hormigón, en forma de probetas paralepipédicas, y midiendo el número y separación de las fisuras. Los ensayos se realizaron a $-160{ }^{\circ} \mathrm{C}$ y a temperatura ambiente, para comparar. A igualdad de fisuración global se prefiere, en general, muchas fisuras pequeñas y juntas que pocas grandes y separadas. Los resultados indicaron que al disminuir la temperatura aumenta la separación máxima entre fisuras, pudiendo aumentar hasta un $80 \%$ a $-160{ }^{\circ} \mathrm{C}$. Otra conclusión que parece desprenderse de estos ensayos es que la relación $\sigma_{T} / \tau$, resistencia a tracción del hormigón y tensión de adherencia crece al disminuir la temperatura, ya que puede supo- nerse que dicha separación es proporcional a $\sigma_{T} / \tau$, puesto que de forma aproximada:

$$
\sigma_{T} \mathrm{~A}=\pi \mathrm{D} \int_{0}^{L / 2} \tau^{\prime} \mathrm{dL}=\frac{1}{2} \pi \mathrm{DL} \tau
$$

de donde,

$$
\mathrm{L}=\frac{2 \mathrm{~A}}{\pi \mathrm{D}} \cdot \frac{\sigma_{T}}{\tau}
$$

donde $A$ es el área del hormigón, $D$ el diámetro de la armadura, $L$ la separación entre fisuras, $\tau^{\prime}$ el valor de la adherencia en cada punto y $\tau$ el valor medio.

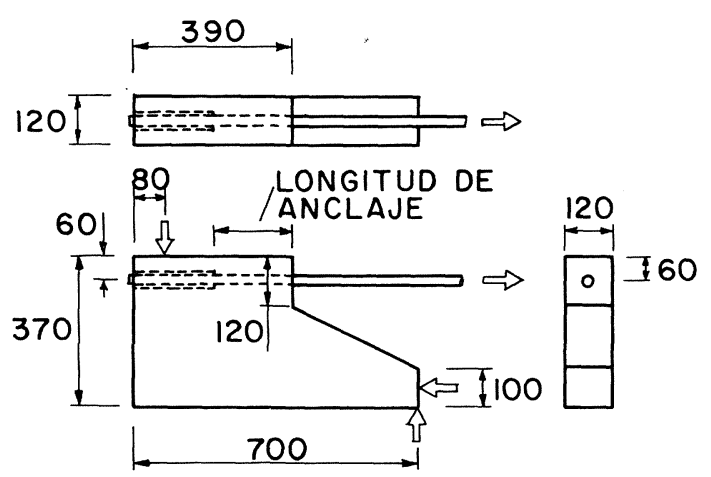

ESQUEMA DE LA PROBETA

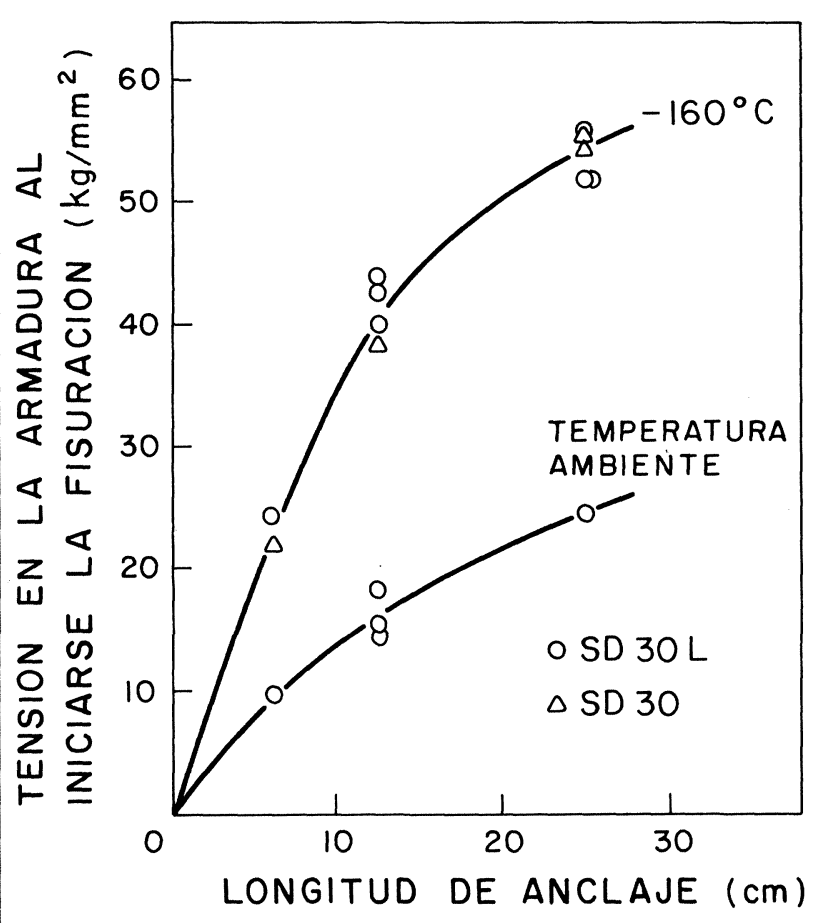

Fig. 12. - Ensayos de adherencia a temperatura ambiente y a $-160^{\circ} \mathrm{C}$. Las curvas corresponden al inicio de la fisuración del hormigón. 
Y. Goto y Miura también realizaron otros ensayos para estudiar la capacidad de transmisión de esfuerzos en los empalmes y anclajes. Para ello hicieron dos series de pruebas con probetas similares a la esquematizada en la figura 12 . En la primera serie primero se fisuró el hormigón del recubrimiento determinando asi una cierta tensión de adherencia. En la segunda serie se provocó primero el deslizamient de la armadura mediante un adecuado refuerzo del hormigón. En este ensayo no se midió el desplazamiento relativo no pudiendo estimar, por lo tanto, la curva tensión de adherencia-desplazamiento. En las dos series se observó un incremento del correspondiente valor de la tensión de adherencia, alrededor de 2,5. Los resultados de la primera serie también se han representado en la figura 12 .

Para estudiar la transmisión de los esfuerzos por solapo, los citados autores experimentaron con probetas como las esquematizadas en la figura 13, donde se resumen los resultados, representando la resistencia relativa a los valores de la temperatura ambiente en función de la longitud de solapo. Parece ser que el espesor del recubrimiento no influye mucho y que la resistencia tiende a disminuir con la longitud del solapo.

La influencia de la humedad en el hormigón, de la relación agua/cemento y de los aireantes en la adherencia a bajas temperaturas, las han estudiado S. Yamane, M. Kasami y T. Okuno en ensayos de arrancamiento con probetas cúbicas de $15 \mathrm{~cm}$ de lado. Los resultado de los ensayos indican que la adherencia entre el hormigón y la armadura (lisa, de $19 \mathrm{~mm}$ de diámetro) aumenta mucho con la disminución de la temperatura para hormigones saturados, llegando a ser a $-196{ }^{\circ} \mathrm{C}, 5,8$ veces superior al valor a temperatura ambiente. En probetas curadas con una humedad relativa del $45 \%$ este valor solamente llega a 1,4.

En otra serie de ensayos hasta $-70{ }^{\circ} \mathrm{C}$ con probetas saturadas, con relaciones agua/cemento de $50 \%, 60 \%$ y $70 \%$, contenido en aire del $2 \%$ y dos tipos de armaduras (lisas y corrugadas) se detectó un aumento de la adherencia con la disminución de la temperatura, alcanzándose valores del doble, aproximadamente, de los de la temperatura ambiente. Unicamente una serie, con armadura lisa, relación agua/cemento del $70 \%$ y un contenido en aire del 6,7 \% alcanzó una resistencia 5 veces superior al valor de la temperatura ambiente.

Estos resultados muestran que el hormigón armado se comporta de forma diferente a bajas temperaturas y que es necesario investigar más si se quiere predecir su comportamiento cuando nos alejamos de la temperatura ambiente.

\section{4.--Hormigón pretensado}

El hormigón pretensado es uno de los pocos materiales utilizados a temperatura ambiente que se comporta satisfactoriamente a bajas temperaturas. Desde los años cincuenta, cuando se construyeron los primeros depósitos para almacenar oxígeno líquido, hasta nuestros dias, donde depósitos de más de $100.000 \mathrm{~m}^{3}$ almacenan gas natural licuado, numerosos recipientes de hormigón pretensado atestiguan la idoneidad de este tipo de estructuras (A. Bruggeling, 1979, F. H. Turner, 1979, M. Elices, 1981). No obstante, todavia quedan aspectos del comportamiento del hormigón que necesitan conocerse mejor. Además de los ya mencionados, el comportamiento del hormigón en las zonas de anclaje de las armaduras activas y los

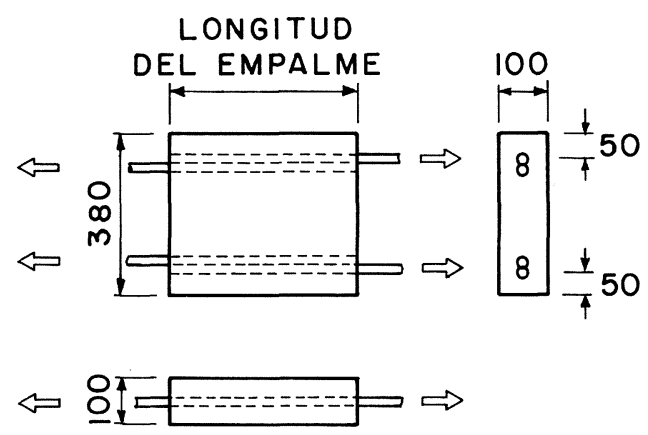

ESQUEMA DE LA PROBETA

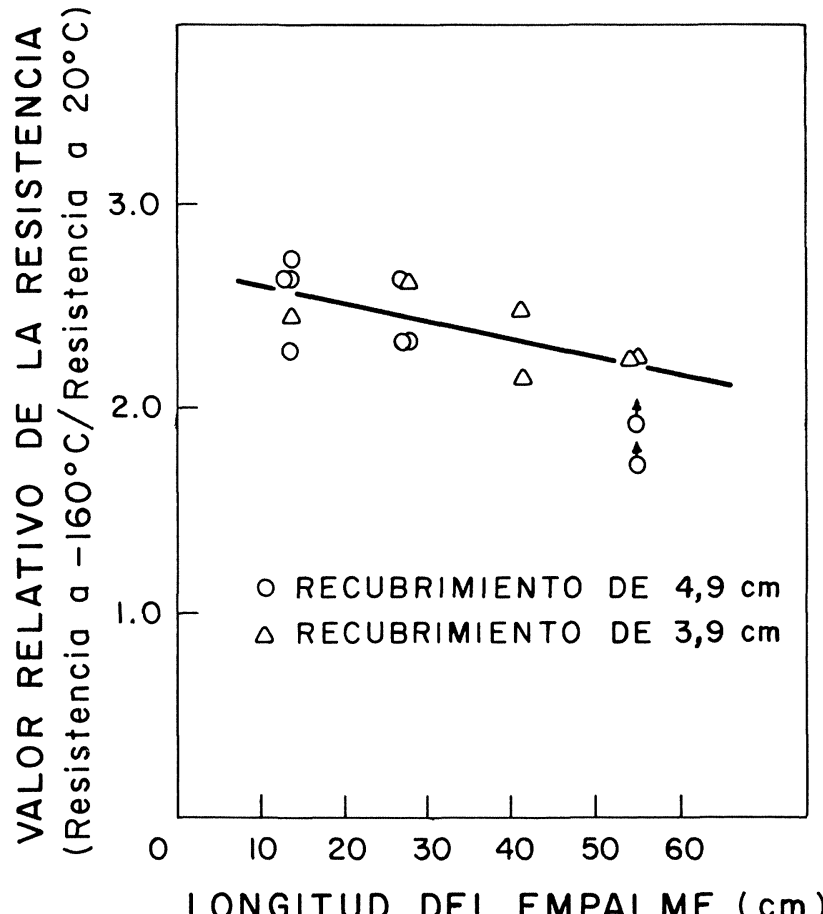

Fig. 13. - Ensayos de adherencia. Influencia de la longitud del empalme por solapo y del espesor del recubrimiento. 
fenómenos diferidos, como la fluencia y la conducción térmica, al estar más relacionados con el hormigón pretensado se han agregado en este último capítulo.

La información sobre el comportamiento del hormigón a bajas temperaturas en las zonas de los anclajes es prácticamente nula. En los elementos pretensados donde el anclaje se produce por adherencia, no se ha estudiado esta propiedad, probablemente porque este tipo de anclaje no se ha utilizado. Tampoco se ha estudiado la adherencia del tendón con la lechada en los sistemas postesados, ni el comportamiento mecánico del hormigón bajo las solicitaciones complejas que se producen alrededor de los anclajes. Unicamente se han llevado a cabo ensayos de recepción, bastante sencillos (M. Elices, J. Planas, et al 1979) que indican un comportamiento satisfactorio del hormigón en estas circunstancias. Los sistemas de anclaje junto con los tendones se han comentado en otros trabajos (M. Elices 1978, M. Elices y G. Sleigh 1978, M. Elices, F. Rostasy y W. Faas 1982).

En un elemento pretensado las deformaciones diferidas del hormigón, ya sean debidas a esfuerzos mecánicos, fluencia, o a variaciones de temperatura debidas a la transmisión de calor, puedén originar importantes variaciones en el estado tensional justificando con ello los siguientes apartados.

\subsection{Fluencia del hormigón}

La fluencia del hormigón a bajas temperaturas la han medido M. Aoyagi y M. Kawasaki (1973) y posteriormente T. Okada y M. Iguro (1978) en una serie de ensayos más completa orientada al estudio de vigas pretensadas. Este resumen está basado en el segundo trabajo.

Antes de estudiar la fluencia en vigas de hormigón, T. Okada y $M$. Iguro midieron en una serie de experimentos la flexibilidad de varias vigas pretensadas para comprobar la capacidad de predicción a bajas temperaturas utilizando las expresio nes descritas anteriormente en 2.2 y 2.3 para la resistencia del hormigón. Los resultados se indican en la Figura 14 y en la Tabla 3 . Como puede apreciarse, tanto la rigidez como la capacidad de deformación se incrementaron a $-70{ }^{\circ} \mathrm{C}$. Las pre dicciones para la carga de fisuración y la carga máxima están en buen acuerdo con los resultados experimentales.

Posteriormente se midió la fluencia en el hormigón y, a partir de estos resultados, se predijo el comportamiento de las vigas pretensadas sometidas a un gradiente térmico y distintas solicitaciones.

Para determinar el coeficiente de fluencia se hicieron ensayos uniaxiles de compresión, con probe-

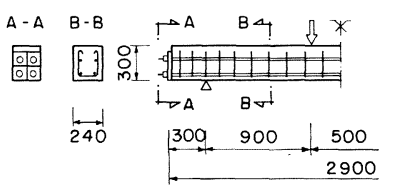

ESQUEMA DE LA VIGA

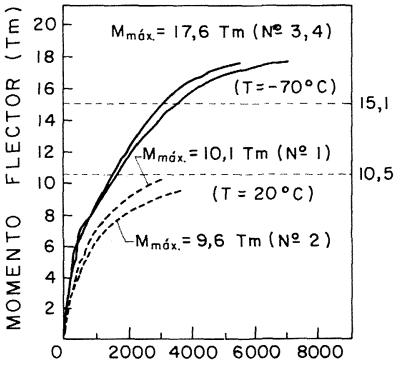

MAXIMA DEFORMACION DEL HORMIGON A COMPRESION $\left(\times 10^{-6}\right)$

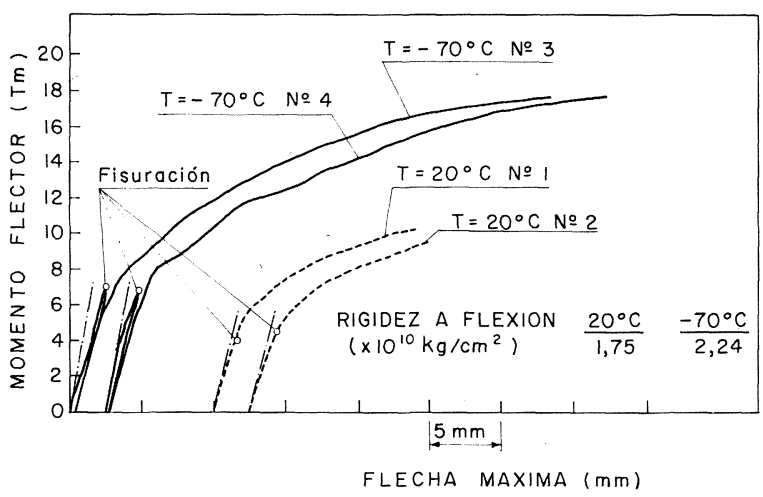

Fig. 14. --Ensayos de flexión en vigas pretensadás.

tas saturadas de 75 dias de edad, a $20^{\circ} \mathrm{C},-10^{\circ} \mathrm{C}$ y $-30^{\circ} \mathrm{C}$. Los ensayos duraron 87 y 175 dias. La diferencia entre los ensayos $\mathrm{a}-10{ }^{\circ} \mathrm{C}$ y a $-30{ }^{\circ} \mathrm{C}$ no es grande, lo cual está de acuerdo con los resultados de Aoyagi que indica que las deformaciones por fluencia a $-80^{\circ} \mathrm{C}$ son similares a las observadas $\mathrm{a}-20^{\circ} \mathrm{C}$. Los resultados se muestran en la figura 15 donde se ha representado, también, a temperatura ambiente, la predicción según el documento CEB-FIP, State of Art Report 1973. Los valores experimentales a bajas temperaturas se pueden ajustar a la siguiente expresión:

$$
\psi(t)=0,086 \log t+0,019
$$

donde $\psi(t)$ es el coeficiente de fluencia al cabo de $t$ horas (t $>1$ hora) y para un intervalo de temperaturas entre $-10^{\circ} \mathrm{C}$ y $-30{ }^{\circ} \mathrm{C}$. La deformación, al cabo de $t$ horas, viene dada por la expresión usual:

$$
\varepsilon(t)=\psi(t) \frac{\sigma}{E}
$$

donde el módulo de elasticidad a la temperatura correspondiente se puede estimar a partir de la fórmula dada al final de 2.3. Otra forma de estimar la fluencia en el intervalo de temperaturas citado se basa en la observación hecha por Okada de que la deformación es aproximadamente la mitad de la correspondiente a temperatura ambiente. 
TABLA 3. ENSAYOS DE FLEXION EN VIGAS PRETENSADAS

\begin{tabular}{cccccc}
\hline \multirow{2}{*}{ Ensayo } & & \multicolumn{2}{c}{ Calculado } & \multicolumn{2}{c}{ Medido } \\
& & $\mathbf{M}_{\text {fis }}$ & $\mathbf{M}_{\max }$ & $\mathbf{M}_{\text {fis }}$ & $\mathbf{M}_{\max }$ \\
\hline Temperatura $+20^{\circ} \mathrm{C}$ & Viga 1 & \multirow{2}{*}{4,7} & 10,5 & 4,1 & 10,1 \\
& Viga 2 & & & 4,5 & 9,6 \\
\hline Temperatura $-70^{\circ} \mathrm{C}$ & Viga 3 & \multirow{2}{*}{8,9} & 15,1 & 7,0 & 17,6 \\
& Viga 4 & & & 6,8 & 17,6 \\
\hline
\end{tabular}

$M_{\text {fis. }}$ Momento que inicia la fisuración (t.m.).

$\mathrm{M}_{\max }$. Momento máximo.

Los resultados obtenidos dividiendo por dos la curva CEB-FIP también se han indicado en la figura 15.

Finalmente, Okada e Iguro realizaron ensayos de 1000 horas de duración para comprobar las predicciones teóricas.

Las vigas pretensadas se ensayaron a la edad de 90 dias, sometidas al gradiente térmico indicado esquemáticamente en la figura 16 y a las solicitaciones siguientes:

a) En el primer experimento, la solicitación exterior se fue variando con el tiempo de forma que la deformación de la viga, debida al gradiente térmico y a la fluencia, fuera nula. En la figura $16 a$ se ha representado la variación del esfuerzo en el tensor en función del tiempo.

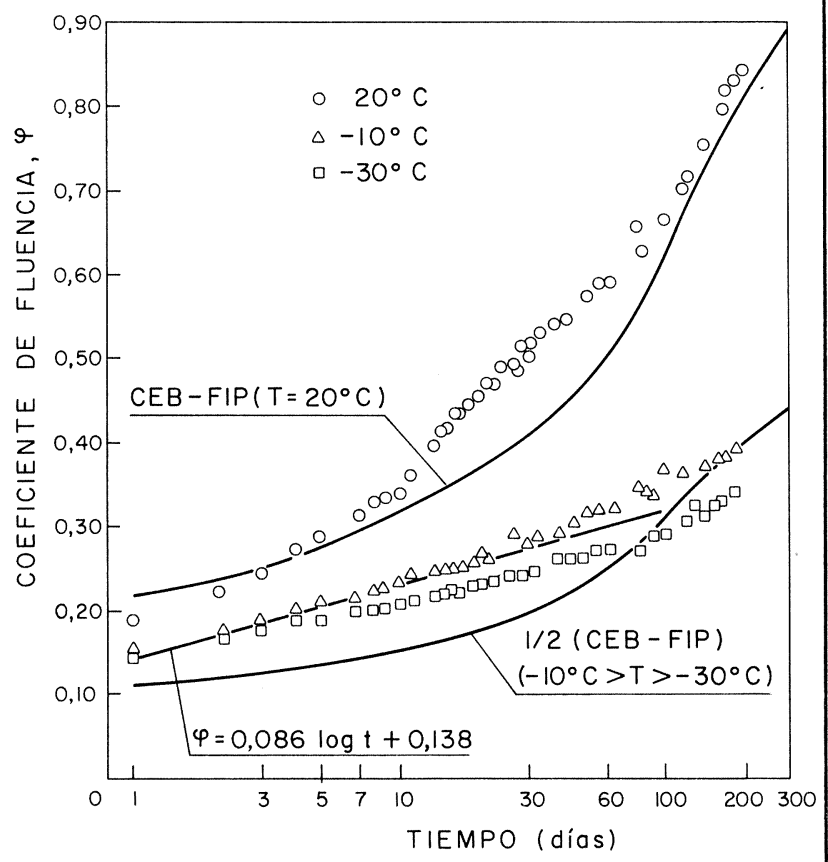

Fig. 15.--Influencia de la temperatura en el coeficiente de fluencia de hormigón saturado.
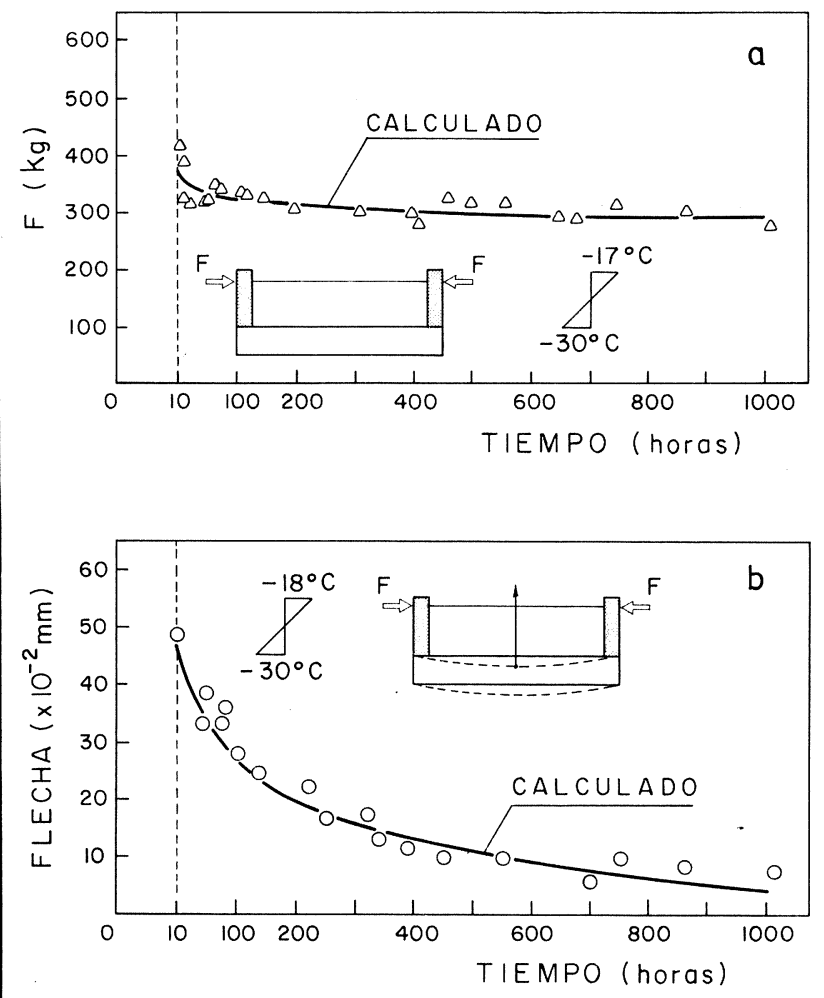

Fig. 16. --Deformaciones diferidas en vigas pretensadas, debidas a un gradiente térmico y a la fluencia del hormigón.

b) En un segundo experimento la solicitación exterior se mantuvo constante y se registró la flecha en el centro, debida al gradiente térmico y a la fluencia, en función del tiempo, figura 16b.

Ambos resultados concuerdan bastante bien con las predicciones teóricas utilizando las expresiones que se citan en el artículo.

\subsection{Conductividad térmica y calor especifico del hormigón}

Para algunas hipótesis de cálculo hace falta conocer la distribución de temperaturas para poder cal- 
cular las tensiones de origen térmico. Para realizar estos cálculos hacen falta valores de la conductividad térmica, $\lambda, y$ del calor específico, $c$, en función de la temperatura, de la dosificación del hormigón y de la humedad del mismo. Nuevamente nos encontramos con falta de datos y de experimentación, sólo se han publicado algunos resultados como consecuencia de la investigación realizada en los laboratorios de la Portland Cement Association por encargo de Institute of Gas Technology (A. Lentz y G. Monfore 1965, 1966).

La conductividad térmica del hormigón depende bastante de la humedad del mismo, aumentando al aumentar la humedad. El hormigón seco tiene una conductividad baja debido a la poca conductividad del aire contenido en los poros. En la figura 17 se representa la conductividad relativa a la temperatura ambiente (para un hormigón almacenado a $24{ }^{\circ} \mathrm{C}$ y humedad relativa del $50 \%$ ), en función de la temperatura y para distintas condiciones de humedad (M. Reinhardt, 1979).

El calor especifico del hormigón depende de su naturaleza, dosificación y humedad. A temperatura ambiente varia entre 0,85 y $1,15 \mathrm{~J} / \mathrm{g}{ }^{\circ} \mathrm{C}$. Aunque no se dispone de datos a bajas temperaturas no cabe esperar grandes variaciones en este parámetro.

Al enfriar un elemento pretensado pueden aparecer, transitoriamente, tensiones debidas a la diferencia de temperaturas entre el hormigón y las armaduras. Esta situación puede producirse por diferencias en la conductividad térmica, por la geometria y distribución de los tendones o, simplemente, porque haya vias de acceso más rápidas (puentes de frío), como, fisuras, uniones, etc. Durante el estudio experimental $a-165^{\circ} \mathrm{C}$ de un sistema tendón-anclaje (M. Elices et al 1979) se

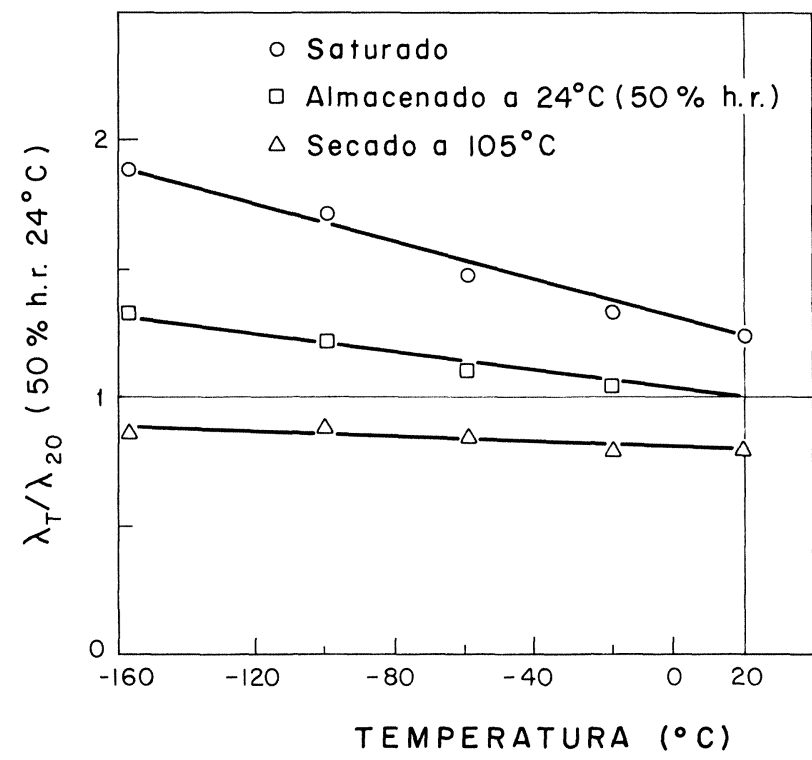

Fig. 17.--Conductividad térmica del hormigón en función de la temperatura y del contenido en humedad.

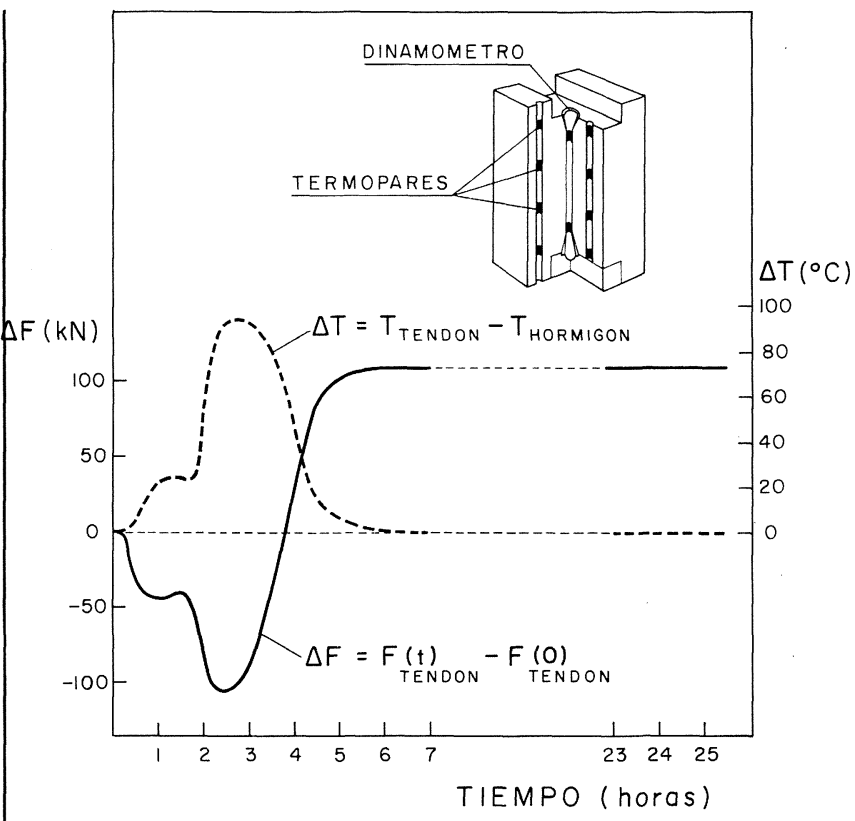

Fig. 18. --Variación de la fuerza del tendón durante el enfriamiento diferencial en un elemento pretensado.

observó este fenómeno. En la figura $18^{\star}$ se indica, de forma esquemática, el elemento pretensado, la disposición del tendón y la situación de los termopares. Al principio, durante el enfriamiento, el hormigón se enfrió más rápidamente que el tendón (en la figura se ha representado la evolución con el tiempo de la diferencia de temperaturas medias entre el tendón y el hormigón). Simultáneamente se registró el esfuerzo en el tendón, mediante un anillo dinamométrico, observándose una pérdida de tensión de 10 toneladas coincidiendo con la máxima diferencia de temperaturas. Finalmente, cuando se uniformó la temperatura se detectó un retesado estable del tendón, de 10,5 toneladas, producido por la diferencia entre los coeficientes de dilatación del acero y del hormigón. Estos resultados ponen de relieve la importancia de los fenómenos transitorios y la necesidad de conocer las caracteristicas térmicas de los materiales para calcular la respuesta de la estructura bajo determinadas hipótesis del proyecto.

\section{5.--Conclusiones}

El propósito de esta sintesis ha sido ofrecer una visión panorámica del estado actual de los conocimientos sobre el comportamiento del hormigón por debajo de $0{ }^{\circ} \mathrm{C}$ hasta la temperatura del nitrógeno líquido.

El proyectista dispone de expresiones que le permiten estimar la respuesta del hormigón cuando se le solicita a bajas temperaturas. Hemos visto cómo se habian hecho buenas predicciones para la resistencia de probetas, para ensayos de flexión de vigas y hasta de fluencia combinada con una 
Informes de la Construcción/332

distribución no uniforme de temperaturas. Además, no siempre se está del lado de la seguridad, se han descrito situaciones - como la fatiga térmica en presencia de mucha humedad o un enfriamiento brusco- donde la respuesta es desfavorable. Ahora ya se pueden prever algunas situaciones y tomar en determinados casos las medidas oportunas.

El constructor puede utilizar criterios para valorar qué parámetros debe controlar mejor durante la construcción y qué tipo de ensayos especificos debe realizar. La humedad desempeña un papel importante y es probable que su distribución en la estructura no sea uniforme. Una juiciosa elección de las variables a ensayar aumentará la seguridad de la obra. Como estos ensayos no están normalizados es preciso tener cuidado cuando se comparan resultados procedentes de distintos laboratorios y también al decidir las condiciones del ensayo más idóneas.

Finalmente, el científico es más consciente de aquellas áreas que necesitan experimentación y estudio. El comportamiento del hormigón bajo solicitaciones complejas - estados multiaxiles, efectos dinámicos- apenas se conoce, lo mismo sucede con la fisuración y la fractura, y aunque quedan problemas concretos por explorar, como la permeabilidad al gas natural licuado y otros gases licuados, ya se entrevé que el hormigón, como material estructural, está destinado a desempeñar un importante papel en la tecnología de la criogenia.

\section{Agradecimientos}

El autor desea expresar su agradecimiento a sus colaboradores del Departamento de Física y Física de Materiales y en especial a los Profesores V. Sánchez-Gálvez, J. Planas, H. Corres y A. Mestre por la valiosa colaboración durante la preparación de este trabajo. También desea agradecer a los Profesores F. Rostasy y Y. Goto por sus sugerencias y la información suministrada; asi como al Departamento de Investigación Aplicada de AUXINI, S. A., por el estimulo y las facilidades recibidas.

\section{Bibliografía}

- Besada, J.L., Elices, M., Planas, J., Sánchez-Miñana, J., Garcia J. A. A new technique of deformation measurements based on microwave resonant cavities.

Proceedings European Microwave Conference. Varsovia (1980).
- Browne, R. D. Bamforth, P. B.

The use of concrete for cryogenic storage. A summary of research, past and present.

1st. Int. Conf. Cryogenic Concrete. Newcastle (1981).

- Bruggeling, A.S.G.

Concrete Storage Vessels. Research Report.

Delft University of Technology (1979).

- Corres, H., Planas, J., Elices, M.

Informe técnico. AUXINI, S. A. (1981).

- Eakin, B.E

Below ground storage of liquefied Nature Gas in prestressed concrete

Institute of Gas Technology. Rep. n. ${ }^{\circ} 8$ (1963).

- Elices, M., Sleigh, G.

Steels for prestressed concrete structures for cryogenic applications.

Proceedings $8 .^{\circ}$ Congreso FIP. Parte 2. Londres (1978).

- Elices, M., Sleigh, G.

El acero en estructuras pretensadas para usos criogénicos.

Hormigón y Acero n. ${ }^{\circ}$ 127(97-109) (1978).

- Elices, M., Planas, J., Caballero, L., Morena, J.M., SánchezGálvez, V., Mestre, A., Astiz, M.A., Valiente, A.

Comportamiento a la temperatura del GNL de un sistema tendón-anclaje postesado a $165 \mathrm{~T}$.

Hormigón y Acero n. ${ }^{\circ}$ 130, 131, 132 (85-93) (1979).

- Elices, $M$.

Prestressed cryogenic concrete. A review of testing in Spain. 1st. Int. Conf. Cryogenic Concrete. Newcastle (1981).

- Elices, M., Rostasy, F., Faas, W.M.

Cryogenic behaviour of Materials for prestressed concrete. FIP Report (1982).

- Elices, M., Planas, J.

Measurement of tensile strength of concrete at very low temperatures

Journal of the American Concrete Institute. V. 79 (1982).

- Grubl, P.

Uber die Rolle des Eises im Gefuge zementgebundener Baustoffe.

Beton-Herstellung und Verwendung 31, N. ${ }^{\circ} 2$ (54-58) (1980).

- Goto, Y., Miura, T.

Experimental studies on properties of concrete cooled to about $-160^{\circ} \mathrm{C}$

Tohoku University. Report 44 (357-385) (1979).

- Lentz, A.E., Monfore, G.E.

Thermal conductivity of concrete at very low temperatures. J. PCA. (39-46) (1965).

- Lentz, A.E., Monfore, G.E

Thermal conductivities of Portland cement paste, aggregate and concrete down to very low temperatures.

J. PCA.

- Monfore, G.E., Lentz, A.E.

Physical properties of concrete at very low temperatures. Bull. PCA Research Dept. N14 (33-39) (1962).

- Okada, T., Iguro M.

Bending behaviour of prestressed concrete beams under low temperatures

J. Japan Prestressed Concrete Eng. Ass. (17-25) (1978).

Planas, J Elices, M. Sánchez-Gálvez, V., Mestre $A$

Propiedades mecánicas a temperaturas criogénicas del acero de pretensado.

Hormigón y Acero n. ${ }^{\circ} 126$ (89-99) (1978). 
- Reinhardt H.W.

Mechanische en fysische eigens chappen van beton Cement 31 (7-13) (1979).

- Rostasy F., Schneider, U., Wiedemann, G. Behaviour of mortar and concrete at extremely low temperatures.

Cement and Concrete Research. Vol. 9 (365-376) (1979).

- Rostasy, F., Wiedemann, G. Strength deformation and thermal strains of concrete at cryogenic conditions.

1st. Int. Conf. on Cryogenic Concrete. Newcastle (1981).

- Setzer, M.J.

Einflu $\beta$ des Wassergehalts auf die Eigenschaften von Beton. DAfStb, Heft 280 (1977), Wilh. Ernst u. Sohn, Berlin.
- Stockhausen, N., H. Dorner, B. Zech and M.J. Setzer. Untersuchung von Gefriervorgangen im Zementstein mit Hilfe der DTA.

Cement and Concrete Research Vol. 9, pp. 783-794 (1979).

- Tognon, G.

Behaviour of mortons and concretes in the temperature range from $20^{\circ} \mathrm{C}$ to $-196{ }^{\circ} \mathrm{C}$.

5th. Int. Sympo. Chemistry of Cement (229-249) (1969).

- Turner, F.H.

Concrete and cryogenics.

Cement and Concrete Association (1979).

- Yamane, S., Kasami, H., Okuno, T.

Properties of concrete at very low temperatures ACI SP 55-9 (208-221) (1978).

\section{publicaciones del i.e.t.c.c.}

\section{las resinas epoxi en la construcción}

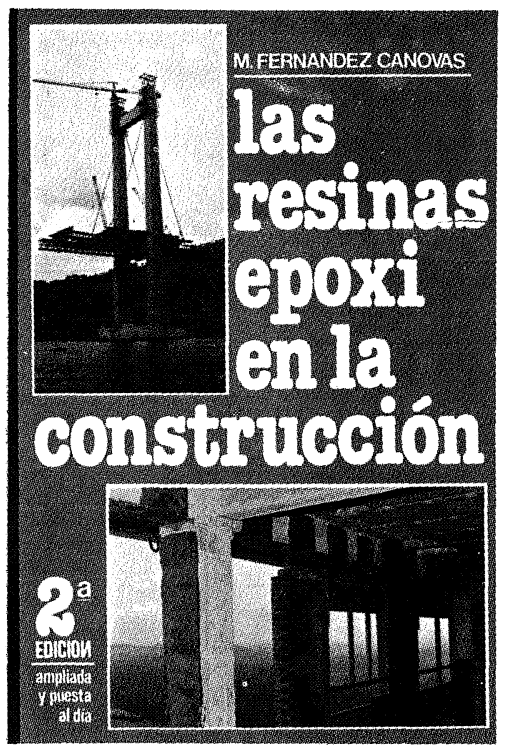

Manuel Fernández Cánovas

Dr. Ingeniero de Construcción

Este libro, el primero en lengua castellana sobre resinas epoxi aplicadas a la construcción, está dirigido a arqui-

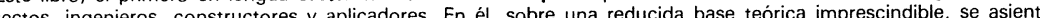
oda una extensa gama de aplicaciones de gran interes.

El autor trabaja desde hace muchos años en el campo de la investigación, especialmente en el estudio de refuerzos

Con un lenguaje sencillo se tocan todos los problemas que pueden presentarse en la construcción y en los que la solución puede radicar en el correcto empleo de las resinas epoxi.

Se estudian los componentes de las formulaciones epoxi, sus propiedades fisicas y químicas, y aplicaciones deteniéndose, detalladamente, en las siguientes:

Unión de hormigón fresco a hormigón endurecido. - Unión de hormigones entre sí.-Inyecciones de fisuras grietas. - Unión de acero a hormigon. - Barnices y pinturas. - Las combinaciones brea-epoxi. - Revestimientos de depositos alimenticios. - Sellado de superficies cerámicas. - Protección de tubos. - Los suelos epoxi en sus diferentes variantes. - Terrazo epoxi. - Reparación de baches. - Reparación de desperfectos en estructuras. - Reparación de carreteras de hormigón. - Juntas elásticas. - Guardacantos de tableros de puentes. - Refuerzos de pilares, vigas, forjados y zapatas, etc. - Consolidación de suelos. - Anclajes. - Protección de aceros en pretensado.

Se termina con unos capitulos dedicados a la limpieza y preparación de las superficies según los materiales a unir al control del estado superficial de éstos; a las condiciones de temperatura de aplicación; limpieza de los útiles de a métodos de ensayo de sistemas y aplicaciones epoxidicas.

Un volumen encuadernado en cartoné plastificado con lomo de tela, de $17 \times 24 \mathrm{~cm}$, compuesto de 334 páginas y 158 figuras y fotografias.

Madrid, 1981.

Precios: España, 1.700 ptas.; extranjero, \$ USA 34.00

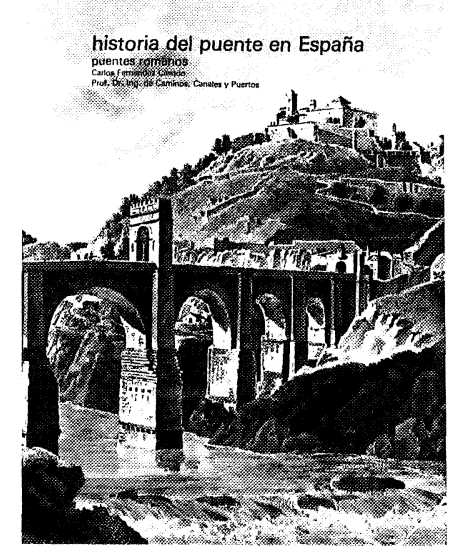

\section{historia del puente en España}

puentes romanos apareciendo durante 25 años en la Revista "Informes de la Construcción", a partir del mes de marzo de 1955. El proposito era mucho más ambicioso pues se trataba de hacer una "Historia de Carlos Fernández Casado puente en Españan, pero hasta el momento actual sólo se ha revisado la época romana, si bien el autor tiene la intención de prolongar la historia hasta cuando sus años de vida le den lugar.

Prof. Dr. Ing de Caminos,

Canales y Puertos Unos apéndices añadidos a los doce articulos originales informan sobre las variaciones experimentadas por algunos puentes más importantes como la del traslado de las ruinas mejor conservadas del puente de Alconetar, que corrio el peligro de quedar sumergido en el embalse due fue detectada, al quedar durante unos dias cortado el curso del Tajo, para realizar el montaje de los desagües de fondo correspondientes a la presa del citado embalse.

Un volumen encuadernado en cuché, a dos colores, de $21 \times 27,5 \mathrm{~cm}$, compuesto de 554 páginas, 105 grabados, 14 dibujos, 753 fotos blanco y negro, 24 fotos color y 110 dibujos de línea.

Madrid, 1981

Precios: España, 3.000 ptas.; extranjero, \$ USA 60

NOTA:

Debido al actual cambio de la peseta, con respecto al \$USA, todos los pedidos de publicaciones del IETcc (sin incluir revistas) que se efectúen a librerias en el extranjero e incluso directos, ptendrán una bonificación del $30 \%$ sobre los precios marcados en dólares. Este descuento será aplicado por los vendedores - hasta nueva orden - a cualquier pedido que se formule fuera de España. 